\title{
Polarisation optics for biomedical and clinical applications: a review
}

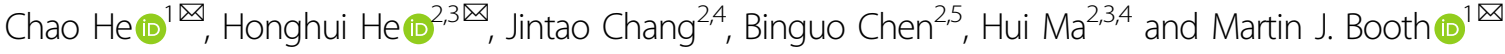

\begin{abstract}
Many polarisation techniques have been harnessed for decades in biological and clinical research, each based upon measurement of the vectorial properties of light or the vectorial transformations imposed on light by objects. Various advanced vector measurement/sensing techniques, physical interpretation methods, and approaches to analyse biomedically relevant information have been developed and harnessed. In this review, we focus mainly on summarising methodologies and applications related to tissue polarimetry, with an emphasis on the adoption of the Stokes-Mueller formalism. Several recent breakthroughs, development trends, and potential multimodal uses in conjunction with other techniques are also presented. The primary goal of the review is to give the reader a general overview in the use of vectorial information that can be obtained by polarisation optics for applications in biomedical and clinical research.
\end{abstract}

\section{Introduction}

Light, as an electromagnetic wave, possesses several fundamental properties, which include intensity, wavelength, phase and polarisation ${ }^{1,2}$ (see Fig. 1a). While the former three are scalar quantities, polarisation has vectorial properties; its use has therefore required more advanced optical and computational approaches. Hence, studies of either the vector properties of light, described via the state of polarisation (SOP) or the full vectorial transformation properties of an object, have a shorter history in biomedical analysis compared with their scalar counterparts, and the extent of their application is still being explored ${ }^{3-5}$. So far, numerous intriguing areas of research have been enhanced through harnessing vectorial information acquired via polarisation optics; these range from fundamental research ${ }^{6-10}$, such as quantified polarisation entropy ${ }^{11}$, across quantum physics ${ }^{12}$, such as

Correspondence: Chao He (chao.he@eng.ox.ac.uk) or Honghui He (he.honghui@sz.tsinghua.edu.cn) or Martin J. Booth (martin.booth@eng.ox.ac.uk) 'Department of Engineering Science, University of Oxford, Parks Road, Oxford OX1 3PJ, UK

${ }^{2}$ Guangdong Engineering Center of Polarisation Imaging and Sensing

Technology, Tsinghua Shenzhen International Graduate School, Tsinghua University, 518055 Shenzhen, China

Full list of author information is available at the end of the article spin-orbital interaction of light ${ }^{13,14}$, to material characterisation (e.g. chiral characteristics ${ }^{15}$ ) or for biomedical studies and clinical applications (e.g. characterisation of structural features in tissue $\mathrm{e}^{16-21}$ ).

Scattering, especially through multiple-scattering processes, alters the degree of polarisation and SOP of the incident light beam ${ }^{22}$. While it is an insightful procedure for evaluating structural information of biomedical samples including tissues and cells ${ }^{16}$, it also introduces uncertainty in expected photon properties ${ }^{22}$. This characteristic largely hinders the development of modern tissue polarimetric techniques and related information analysis $^{20,22,23}$. The turbidity of many tissue structures imposes randomness on the photons' interaction processes, which complicates the detection and analysis of vectorial information ${ }^{20}$. Such phenomena also distinguish tissue polarimetry from the traditional polarisation measurement technique of ellipsometry ${ }^{22-26}$. As summarised in Fig. 1b, their comparison shows several commonalities and differences. The Jones formalism is used for clear and non-depolarising media such as thin films; it consists of the Jones vector (describing the polarisation property of the light) and Jones matrix (describing the polarisation transformation properties of the object). They have been

\section{(c) The Author(s) 2021}

(c) (i) Open Access This article is licensed under a Creative Commons Attribution 4.0 International License, which permits use, sharing, adaptation, distribution and reproduction in any medium or format, as long as you give appropriate credit to the original author(s) and the source, provide a link to the Creative Commons license, and indicate if changes were made. The images or other third party material in this article are included in the article's Creative Commons license, unless indicated otherwise in a credit line to the material. If material is not included in the article's Creative Commons license and your intended use is not permitted by statutory regulation or exceeds the permitted use, you will need to obtain permission directly from the copyright holder. To view a copy of this license, visit http://creativecommons.org/licenses/by/4.0/. 


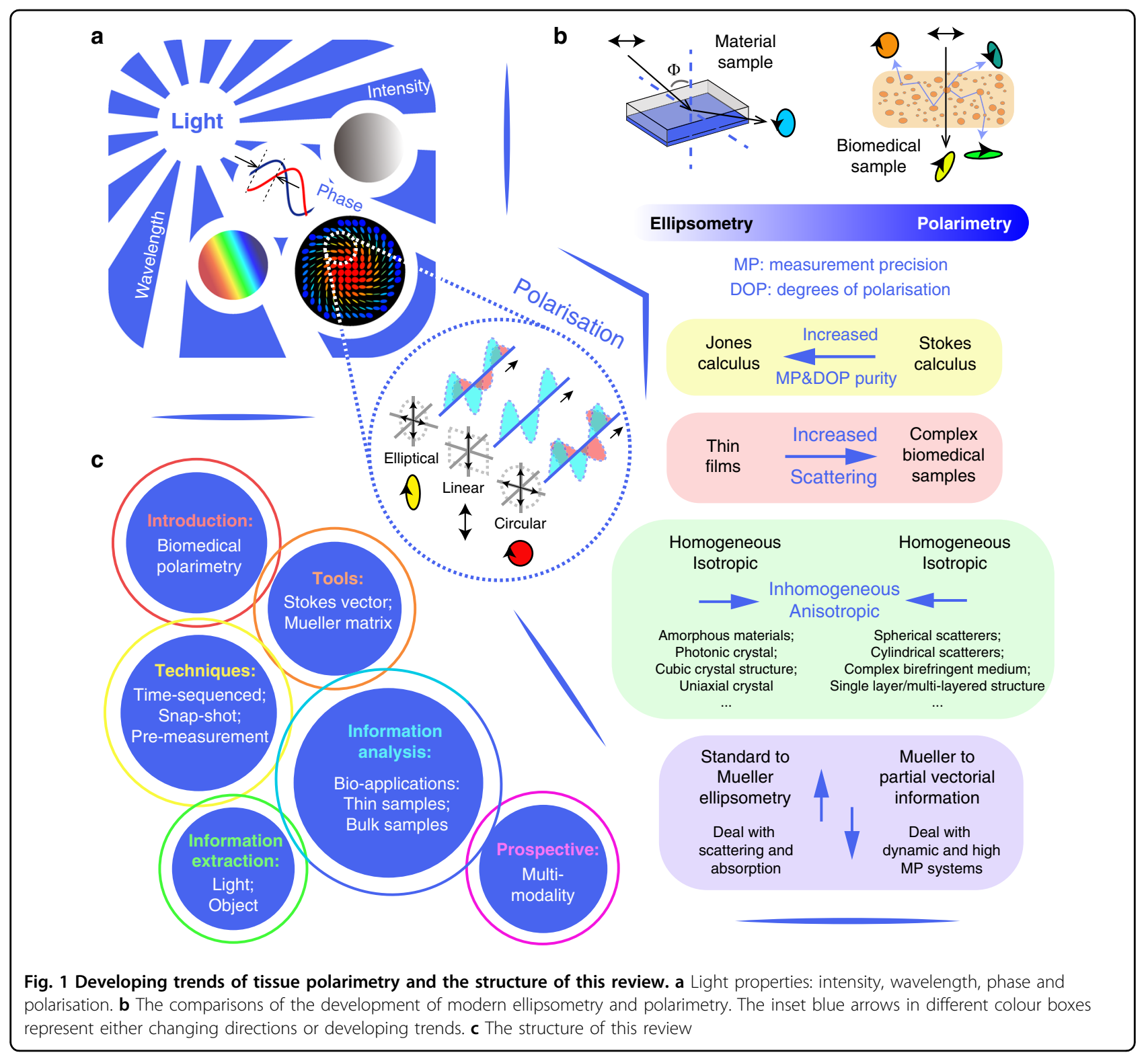

widely used in ellipsometry techniques ${ }^{25,26}$ (see Fig. 1b; and summary in ref. ${ }^{26}$ ). Another polarisation formalism is Stokes-Mueller, in which the Stokes vector and the Mueller matrix are used to describe the light beam and the object, respectively. Neither the Stokes vector nor the Mueller matrix maintain absolute phase information, but have the advantage of being able to represent depolarisation $^{27,28}$. This is often essential in biomedical polarimetry, whose applications normally involve scattering induced light depolarisation ${ }^{20-23}$. There exists an increasing trend in both modern ellipsometry and polarimetry to deal with increasingly complex media, moving from isotropic and homogeneous media towards anisotropic and inhomogeneous ones ${ }^{20-31}$. While modern ellipsometry is developing towards full polarisation measurement using the Stokes-Mueller formalism, advanced polarimetry is gradually changing from full vectorial measurement to partial detection, as some key features of biomedical specimens could possibly be revealed through partial, rather than complete, measurements of vectorial information ${ }^{16,32,33}$.

The structure of this review is given in Fig. 1c; it consists of introducing the basic polarisation optical tools, summarising the current vectorial information detection, extraction, and analysis approaches, and pointing out the possibilities for future multi-modal synergy with other cutting-edge technologies. Although biomedical polarimetry is still developing towards various research fields and applications, largely unexplored spaces still exist. We also hope this review could 
stimulate new explorations or breakthroughs in such prospective fields.

It is worth noting that the use of biomedical polarimetry is expanding and has also been summarised in several recent reviews by Tuchin $^{22}$, Ghosh \& Vitkin ${ }^{20}$, RamellaRoman et al. ${ }^{23}$, Qi \& Elson ${ }^{24}$, De Boer et al. ${ }^{34}$, He et al. ${ }^{16}$. They have demonstrated the fast progress of this technique in the biomedical and clinical fields.

\section{Fundamental vectorial representation for polarised optics in biomedical applications}

Sample induced scattering is prevalent in biomedical imaging, particularly in tissues ${ }^{16,20,22,23}$. This introduces additional SOP modulations that affect diattenuation and retardance as well as depolarisation ${ }^{16,22,23}$. A complex scattering medium can often be modelled by several basic components, like spherical scatterers of different sizes ${ }^{35}$; cylindrical rod-like scatterers with different orientational distributions ${ }^{36}$; and birefringence for an interstitial medium $^{37-39}$; combinations of these features can all be adjusted to mimic the real object ${ }^{36,40-42}$. Other physical conditions such as layer complexity (single-layered or multi-layered scattering ${ }^{43}$ ), or scattering type (elastic domain for Mie and Rayleigh scattering; or inelastic domain, like Raman scattering) are also described in the literature, e.g., see ref. ${ }^{44}$. Modelling of the scattering assumptions can be conducted via Monte Carlo simulation $^{45,46}$. This is a widely used statistical method for quantitative analysis of the interactions between polarised photons and complex biomedical media ${ }^{40-42}$, especially bulk media with multiple-scattering properties, for which the analytical solutions to describe the interactions cannot be obtained. In this review, we focus on the occurrence of elastic scattering in conjunction with other polarisation characteristics (see Fig. 2a) for biomedical polarimetry.

In the presence of depolarisation, Jones calculus, which represents only transitions between pure polarisation states, is of limited use as it cannot comprehensively describe the light properties, especially the degree of polarisation for partially polarised light $t^{2-5}$. Intrinsically, Jones calculus is based on the assumption that electric field vector holds a particular stationary state. For partially polarised light (or fully depolarised light), the variation of the electrical vector as the light propagates is semi-disordered (or completely disordered) so that more degrees of freedom are required to describe the light field ${ }^{47}$. In the scope of linear optics, the Stokes vector, which is a $4 \times 1$ vector, is used to characterise the SOP of the light beam ${ }^{47,48}$; while the Mueller matrix, which is a $4 \times 4$ matrix, describes the transformation properties of the object that affect the Stokes vector ${ }^{47,48}$. Hence, considering that the scope of this review focuses on tissue polarimetry, we place an emphasis on the Stokes-Mueller formalism.

\section{Stokes vector}

The Stokes vector can be expressed with the format shown in Fig. $2 b^{47,48}$; where

$$
\begin{aligned}
& I=I_{0}+I_{90} \\
& Q=I_{0}-I_{90} \\
& U=I_{45}-I_{-45} \\
& V=I_{R}-I_{L}
\end{aligned}
$$

$I_{0}, I_{90}, I_{45}, I_{-45}$ are the projection intensities (different linear components in directions of $0^{\circ}, 90^{\circ}, 45^{\circ},-45^{\circ}$ with respect to the local coordinate system) of a light beam, $I_{L}$ and $I_{R}$ are components of left/right-handed circular polarised light, respectively. Note some other parameters can be defined with components of Stokes vector: degree of polarisation

$$
\mathrm{DOP}=\sqrt{Q^{2}+U^{2}+V^{2}} / I
$$

degree of linear polarisation

$$
\operatorname{DOLP}=\sqrt{Q^{2}+U^{2}} / I
$$

and degree of circular polarisation of light ${ }^{3-5}$.

$$
\mathrm{DOCP}=\sqrt{V^{2}} / I
$$

From the above expressions, we see that the Stokes vector can be calculated via intensity measurements that can be readily performed in an experiment ${ }^{47,48}$. The Jones vector, on the other hand, is defined by amplitude and phase that cannot be directly measured, which is another reason why the Jones approach is less well suited to biomedical polarimetry ${ }^{20-23}$. The intrinsic reason for the existence of depolarisation is due to temporal or spatial averaging ${ }^{16,20-23}$. If an extremely fast and small detector could monitor the vector properties of the light, then it would only detect polarised light. Such averaging properties can also be found in the definition of the Stokes vector $^{47,48}$. Note the definitions of right-handed circular polarised light (clockwise rotation) and left-handed circular polarised light (anticlockwise rotation) are different in optics books and academic communities. It depends on whether the observer 'sees' the light from the source (Convention I), or from the detector (Convention II). Institute of Electrical and Electronics Engineers (IEEE) uses Convention I, so it is also widely used in engineering fields; Quantum physicists also use Convention I, to be consistent with the conventions for representing particle spin states ${ }^{49,50}$. However, for numerous optics books such as Principles of Optics (Born and $\mathrm{Wolf}^{48}$ ) and 
a
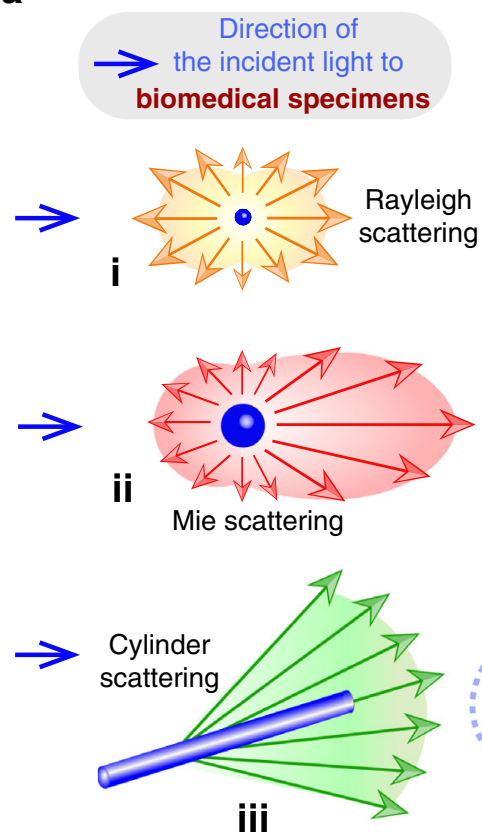

$\underset{\longrightarrow}{\longrightarrow}$

iv

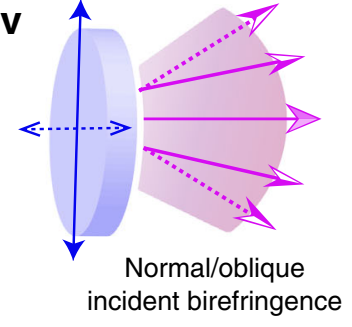

00

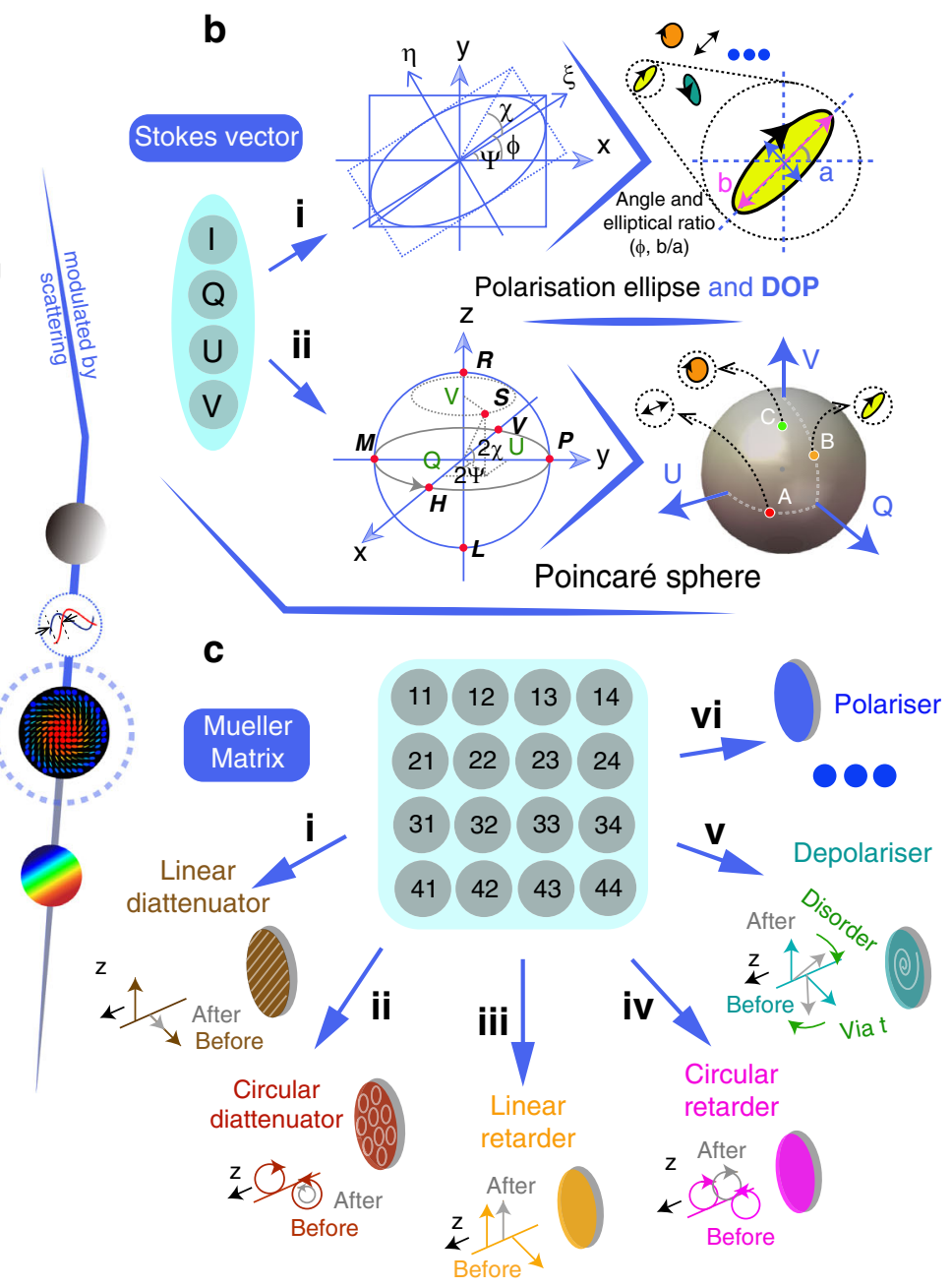

Fig. 2 Fundamental light-matter interaction processes in biomedical specimens and polarisation tools. a Several scattering types and media manipulation, showing schematically the way in which light is scattered in the differing scenarios ${ }^{16-24}$. $\mathbf{b}$ The Stokes vector representations. Top row: the polarisation ellipse and the DOP can be used to represent a given Stokes vector. Bottom row: the Poincaré sphere visualises all states of polarisation with linear states on the equator, circular states on the north/south poles and elliptical states in between ${ }^{2-5}$. $\mathbf{c}$ Vectorial properties that can be encoded in the Mueller matrix. Linear/circular diattenuator, linear/circular retarder etc. are fundamental polarisation elements; the arrows on the lines or circles represent the eigenbasis of modulated light beam passing through such media, where 'before' and 'after' illustrate the amplitude and/or relative phase change of the chosen eigenbasis. For further details refer to refs. ${ }^{2-5}$

Handbook of Optics ${ }^{51}$, Convention II is used. In this review, we use Convention II in order to correspond to such scientific references.

The Jones vector has a graphical representation known as the polarisation ellipse ${ }^{47,48}$ (if we add the parameter DOP, the polarisation ellipse can also represent the Stokes vector (see Fig. 2b (i))). While for Stokes vector visualisation, the Poincaré sphere (PS) is commonly used $^{47,48}$ (see Fig. 2b (ii)). SOPs are represented via the PS, which is defined in a three-dimensional coordinate system, whose coordinates correspond to the eigenbasis formed by $Q, U$ and $V$ (each normalised by $I$ ). The PS is a unitary sphere that represents complete polarisation states on its surface and depolarised states inside the sphere. Any transformation of the SOP through a specimen is equivalent to manipulation of the original Stokes vector between different points on or inside the PS. Figure $2 b$ (ii) gives a schematic demonstration of the PS. The length of the vector from the origin point to the SOP location denotes the $\mathrm{DOP}^{47,48}$. The letters $H, V, M, P$ are specific polarisation states: horizontally polarised $(H)$, vertically polarised $(V), 45^{\circ}$ polarised $(M)$ and $-45^{\circ}$ polarised $(P)$. The polarisation ellipse parameters $(X$ and $\psi$ ) can be interpreted from the azimuth angle (and the polar angle) of the derived vector inside the PS.

Such a graphical representation excludes the absolute phase information, which is sometimes not addressed in typical vectorial beam analysis such as pure polarisation 
measurement or tissue polarimetry ${ }^{20-23,47,48}$. However, one type of the absolute phase variation that is referred to as geometric phase is related to the pathway of the SOP movement on the surface of the $\mathrm{PS}^{47,48}$, which features scope for the extension of the tissue polarimetric technique (also see "Discussion").

\section{Mueller matrix}

The Mueller matrix (MM) describes the vectorial transformation properties of an object ${ }^{16,20-23}$. As illustrated in Fig. 2c, the MM describes the transformation of one Stokes vector into another. The MM represents the full vector properties of an object through its 16 elements $\left(m_{k l} ; k, l=\right.$ $1,2,3,4)$. Among these, $m_{11}$ represents the transformation of scalar intensity (absorption or other loss); the other 15 elements encode the vectorial properties of the object ${ }^{47,48}$. Direct physical meanings of these 15 elements taken individually are normally ambiguous ${ }^{16,20-23}$. As illustrated in Fig. 2c, several fundamental polarisation properties are encoded in (and can be extracted from) the MM. They are linear/circular diattenuation, linear/circular retardance, linear/circular polarisance, linear/circular depolarisation and so on $^{2-5,52-62}$. The effects of each of these fundamental optical mechanisms on the light vectors along the propagation direction $\mathrm{z}$ are shown in Fig. 2c (i) to (vi), where 'before' and 'after' illustrate the amplitude and/or relative phase change of the chosen eigenbasis. Amongst these mechanisms, the diattenuator possesses two different absorption ratios for two polarisation directions; it in effect reduces the intensity of one polarisation compared to the other. The retarder exhibits different refractive indices for two polarised eigenvectors, in effect leading to an additional relative phase difference between the two vectors. The depolariser can modify the DOP of the light beams. For more detailed descriptions of the mechanisms and further examples see refs. ${ }^{2-5}$.

Both Stokes vectors and MMs can represent the effects of time-averaged induced depolarisation ${ }^{16}$. An object may introduce two different classes of depolarisation: homogenous depolarisation and inhomogeneous depolarisation. The former one can lead to a similar DOP change for any SOP; such properties can be observed in media such as a polystyrene sphere solution. The later one can lead to different DOP change for different SOP; typical examples are found in complex biomedical tissue.

Several factors may contribute to depolarisation in experimental scenarios. We describe three main reasons here. (a) The first reason relates to the time domain. In general, the Stokes vector polarimeter is based on intensity measurement ${ }^{26}$, so in practice the intensity recorded at the detector includes a time-integration process. If the SOP changes rapidly, possibly due to multi-scattering induced by complex bio-media, then depolarisation would be measured. (b) This reason relates to the spatial domain.
When imaging processes are involved, every point on the beam section is created through the integration of various sub-beams that could have different polarisation states. The superposition of these states leads to depolarisation. (c) The final reason is given in the spectral domain. Many processes that affect polarisation, such as birefringence and scattering, are also dependent on wavelength. Hence for different wavelengths, variations in amplitude and phase may also lead to depolarisation.

\section{Vectorial information measurement techniques for biomedical applications}

Numerous vectorial information measurement methods have been put forward in the past decades ${ }^{4,7,11,26,28,29,63}$. In this section, we categorise the polarisation measurement techniques into two types: time-sequenced and snap-shot approaches ${ }^{28,29,64-67}$ (see Fig. 3). For both cases, the preparation required before detection is similar and can be divided into three general steps: denoising, optimisation and calibration ${ }^{32,68-78}$ (see Fig. 4). The aim of those steps is to reduce the complex errors that would occur during the measurement process, hence obtaining imaging results with higher precision and accuracy ${ }^{32,69-72}$. The technical aspects of such advanced polarimetry are summarised in the review papers by Azzam ${ }^{28}$, Chipman ${ }^{63}$ and $\mathrm{Tyo}^{79}$.

Before full Stokes vector/MM measurements became widely adopted, there was successful work using fixed input SOP and fixed analysers to perform partial vectorial detection for biomedical applications. Jacques et al. showed crossed-polarised light imaging to enhance surface contrast, detect skin cancer and other lesion margins ${ }^{80,81}$; Demos et al. added the dimension of wavelength based on crossed SOPs ${ }^{82,83}$; Groner et al. noted such techniques can enhance superficial vascular contrast, and hence adopt it into brain perfusion, pancreatic and further clinical diagnoses ${ }^{84}$; Bargo et al. took angle-dependency into consideration when measuring skin tissue ${ }^{85}$. Sridhar et al. also studied multiply scattered photons to enhance information extraction from biological specimens via elliptically polarised light ${ }^{86}$.

Both time-sequenced and snap-shot polarimetry techniques can be classified in two general ways: firstly, as either Stokes vector (light property) or MM (material property) measurement; and secondly, as partial or full vectorial measurement (Fig. 3). We will classify different techniques using the second criteria in later sections of this review.

\section{Time-sequenced techniques}

Stokes polarimetry is clearly the basis for more advanced MM polarimetry. Both of their intrinsic mechanisms can be interpreted with respect to the instrument matrix $(A)^{68-72}$ (see Fig. 4). This matrix 


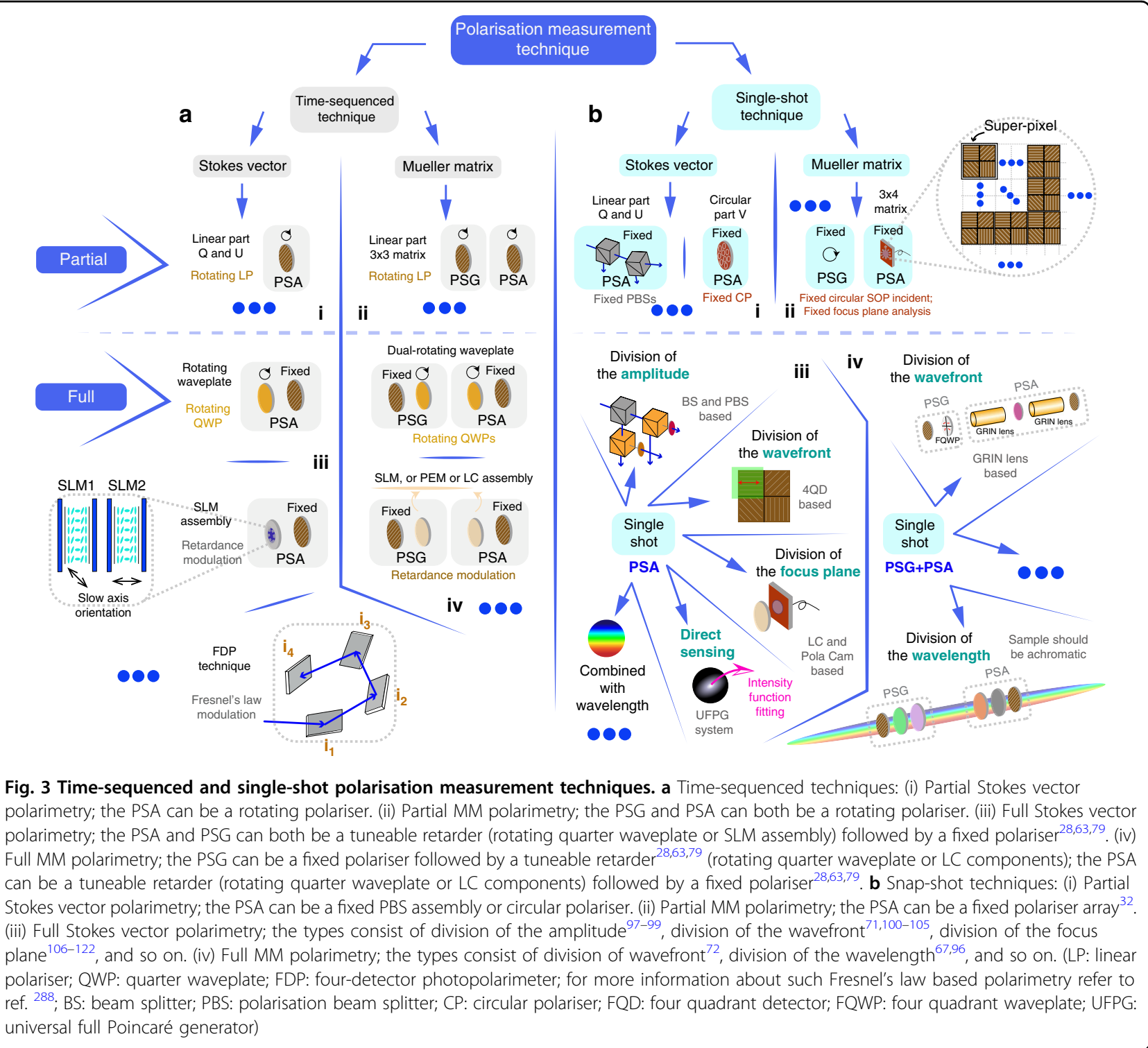

represents the settings of the polarisation state generator (PSG) and polarisation state analyser (PSA) in the various measurement steps: for MM measurement it represents the PSG and PSA, for Stokes vector measurement it represents one the PSA. Combinations of the rotating waveplate and/or polariser are widely adopted in such approaches $^{64-66}$. The original proposal for a Stokes vector measurement scheme (that using SOPs of $H, V, M, P, L$ and $R$ ) was from Collett $^{87}$ in 1984. Later it was adopted for biomedical information extraction or phantom analysis with ability of the full depolarisation information characterisation $^{16,20-23}$.

The use of rotating components has disadvantages, such as increasing measurement time and introducing unexpected errors from mechanical movements. However, such systems are easy to construct. Hence, numerous commercialised polarimeters still use this approach. In order to make improvements, researchers have tried to reduce the number of the rotating components (such as the dual-rotating waveplate MM polarimeter with fixed polarisers that was proposed by Azzam $^{64}$ in 1978 , which is widely used in tissue analysis ${ }^{16,22}$ ) or use fast modulation devices (such as Stokes or MM polarimeters enabled via liquid crystal variable retarders $(\mathrm{LCVR})^{88}$, spatial light modulators $(\mathrm{SLM})^{89}$, ferroelectric liquid crystals $(\mathrm{FLC})^{90}$, or photoelastic modulators $\left.(\mathrm{PEM})^{91}\right)$. Besides full $\mathrm{MM}$ detection, partial MM measurement, such as $3 \times 3$ MM imaging of linear polarisation states, also gained wide attention. Qi et al. used related methods in analysing linear depolarisation and retardance of rat tissue ${ }^{92}$. 


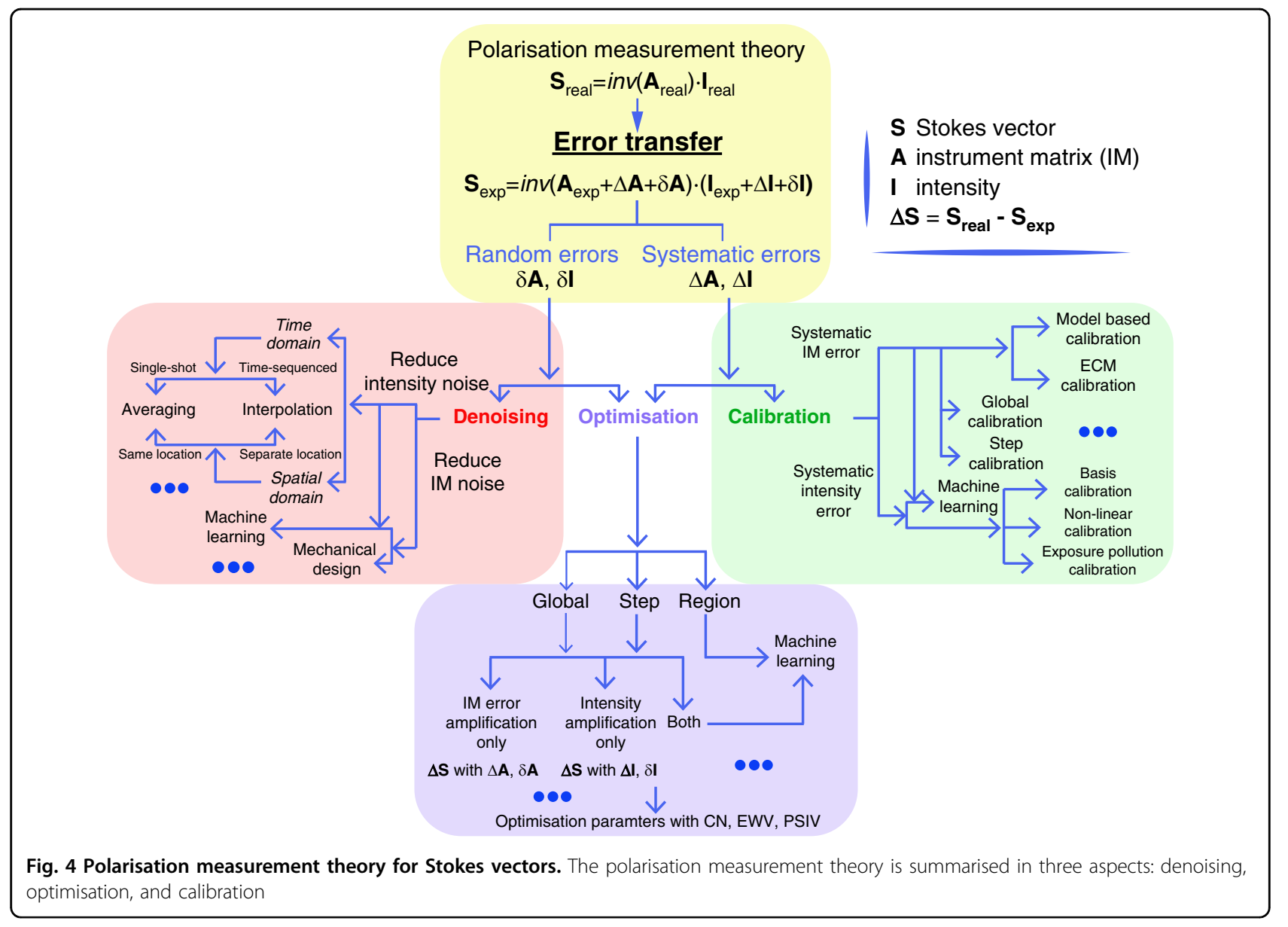

Although there are some applications that require highspeed operation, such as detection in dynamic situations like in vivo sensing for clinical diagnosis ${ }^{24}$, timesequenced polarimeters still play an important role in modern polarimetric research, due to their mature state of development and simple configuration. Such applications include characterisation of complex vector fields ${ }^{6,7,9}$, or providing ground truth validation in tissue research (e.g., differentiating human breast cancer ${ }^{93-95}$ ).

\section{Snap-shot techniques}

Rapidly changing or dynamic objects need snap-shot detection, in order to correctly extract vectorial information that would be complicated by time-sequenced measurement. Snap-shot approaches are configured to take different measurements in parallel, as opposed to the serial measurement of sequential techniques. In general, snapshot techniques must, to some degree, sacrifice alternative dimensions to enable simultaneous vector measurement ${ }^{67,96}$. Those methods include (see Fig. 3b): Stokes vector polarimeters with division-of-amplitude ${ }^{97-99}$, division-of-wavefront ${ }^{71,100-105}$ or division-of-focusplane $\mathrm{e}^{106-122}$ - these fit in the category of spatial modulation with respect to different analysis channels (see Fig. 3b (iii)). Savart-plate-based polarimeters (Oka et al.) are in the category of Fourier frequency domain segmentation, which are interferometric systems where the polarisation information is encoded in the spatial carrier fringes ${ }^{123}$. If combined with the property of birefringence dispersion, spectroscopic polarimetry with channelled spectrum can also be presented ${ }^{67,96}$.

Similar to Stokes vector polarimeters, there exist concepts for snap-shot MM polarimeters, in which certain dimensions are sacrificed to enable simultaneous MM estimation (see Fig. 3b (iv)). Dubreuil et al. ${ }^{67}$ and Hagen et al. ${ }^{96}$ utilised different wavelength-dependent birefringent media to resolve the MM in a single shot, within the limitation of the sample being achromatic. Piquero et al. ${ }^{124}$ utilised full Poincaré beams as a PSG, enabling MM polarimetry with the division of the wavefront. He et al. applied a spatially segmented method with defocusing to measure statistically averaged properties of biomedical samples ${ }^{72}$. As complete snap-shot MM techniques are rather complex, their usage for extractions of biomedical information is less common than the use of single-shot partial MM or Stokes vector polarimetry. For 
instance, $3 \times 4 \mathrm{MM}$ imaging is also gaining attention using circularly polarised illumination ${ }^{32}$; Chang et al. brought such a technique into the human liver and cervical carcinoma tissue analysis ${ }^{32}$ (see Fig. 3b (ii)).

\section{Denoising, optimisation and calibration}

The measurement precision and sensitivity are vital in polarimetric techniques, hence the errors need to be properly controlled ${ }^{28,32,68-78,125}$. However, as Stokes vector or MM measurements belong to high dimensional information detection with multiple components ${ }^{64,66,126}$, the error sources and error transfer process (such as accumulated amplifications through matrix calculations) are very complicated. Several previous analyses can be found in refs. ${ }^{28,29,32,68-78,125-128}$. In Fig. 4, we summarise in one diagram an overview of the structure of the 'denoising, optimisation, and calibration' processes in polarimetric techniques with respect to random errors $(\delta \mathbf{A}, \delta \mathbf{I})$ and systematic errors $(\Delta \mathbf{A}, \Delta \mathbf{I})$. It also can be seen in the figure that three directions towards obtaining the correct vectorial measurements are still developing.

Note again that $\mathbf{A}$ is the instrument matrix for polarimetric measurement specifically, which is determined by the systematic configurations of polarisation optics and determines the error propagation amplification $^{20-24,69-72}$; while I refers to the recorded intensity information. In Fig. 4, we take Stokes vector measurement equation $(\mathbf{S}=\operatorname{inv}(\mathbf{A}) \cdot \mathbf{I})^{20-24,69-72}$ as a main illustration, to show the relationships between three steps in a picture, for simplicity. A similar structure (using the generalised equation: $\left.\mathbf{M}=\operatorname{inv}\left(\mathbf{A}^{\prime}\right) \cdot \mathbf{I}\right)$ can be derived for the MM measurement, which is based fundamentally on the Stokes vector measurement process.

In order to reduce $\delta \mathbf{A}$ and $\delta \mathbf{I}^{32,68-78}$, a 'denoising process' is adopted. Figure 4 shows the approaches in time or spatial domain including time average and interpretation methods. To deal with the $\Delta \mathbf{A}$ and $\Delta \mathbf{I}$, a 'calibration process' is required. Numerous polarimetric calibration methods have been proposed ${ }^{23,29,63,71,129}$; these can be divided into global and local calibration approaches. Note that the calibration process itself also suffers from the error transfer process. Hence, determining the SOPs for calibration, choosing the standard calibration samples, as well as designing specific calibration methods for different systems should be taken into consideration ${ }^{130,131}$. Figure 4 also shows the process of 'optimisation', which can deal with both types of errors, through global and local optimisation approaches. For this process, different evaluation standards have been put forward to estimate the systematic performance. Marenko et al. considered the condition number $(\mathrm{CN})$ in polarimetric optimisation ${ }^{132}$, Ambirajan, Tyo and others have analysed $\mathrm{CN}$-based optimisation on different phantoms ${ }^{70,133-137}$; and Sabatke et al. introduced equally weighted variance
$(E W V)^{69}$ into the polarimetric area; Azzam et al. and following researchers explained the usage of geometry optimisation based on Poincaré sphere internal volume (PSIV $)^{138-140}$. Other useful criteria have also been proposed $^{141,142}$. Such optimisation parameters can be used for evaluating the intrinsic error amplification of a polarimetry, which affect the accuracy and precision of the measurement ${ }^{23,29,63,71,129-131}$. If we consider the $\mathrm{CN}$, the minimum $\mathrm{CN}$ value for a matrix-based Stokes polarimetry is $\sqrt{3}$, which is the theoretical limit for systematic error amplification ${ }^{68,70}$, as opposed to the minimum possible $\mathrm{CN}$ value $(\mathrm{CN}=1)$ for matrix inversion. A similar error amplification also exists in MM polarimetry ${ }^{143}$. The three above-mentioned processes (denoising, optimisation, calibration) are vital for any biomedical polarimetry, as they determine the credibility of the information extraction and further analysis.

For the matrix-based calculation of Stokes polarimetry (within the scope of the above explanations), there exist two problems: first, the mathematical aspect of minimal error amplification through the matrix calculation; second, the practical aspect that the above-mentioned three separate procedures contribute to error accumulation separately, as they require different evaluation criteria and are normally based upon different assumptions. In fact, there exists the possibility to jump out of the domain of matrix calculation for Stokes polarimetry, circumventing those drawbacks. An interesting direction is the adoption of a full Poincaré beam, taking advantage of its feature that maps all SOPs in a single beam ${ }^{144}$. Vella, Zimmerman, He and others have proposed different measurement approaches harnessing such beams based on different phantoms such as stress engineered optics ${ }^{145-151}$ and graded index optics ${ }^{68}$. The full Poincaré beam Stokes vector technique has recently made it possible to have a clear information-based learning approach (such as the task of searching for the brightest points), combining the 'end to end' solution (a combination of above three processes-denoising, optimisation and calibration) together for an enhanced polarimetric measurement precision and accuracy $^{68}$. In essence, this approach means that the Stokes vector retrieval process changes from matrix-based calculation to information-based image processing.

\section{Vectorial information extraction methods for biomedical applications}

Information about the vectorial properties of a biological specimen can be derived partially from the polarisation properties of the light beam or, in a more complete fashion, from the polarisation properties of the tissue itself $^{20-23,152}$. To extract information from the measured Stokes vector or MM (or part of them), different decomposition methods and parameters were proposed to represent meaningful physical processes, to 


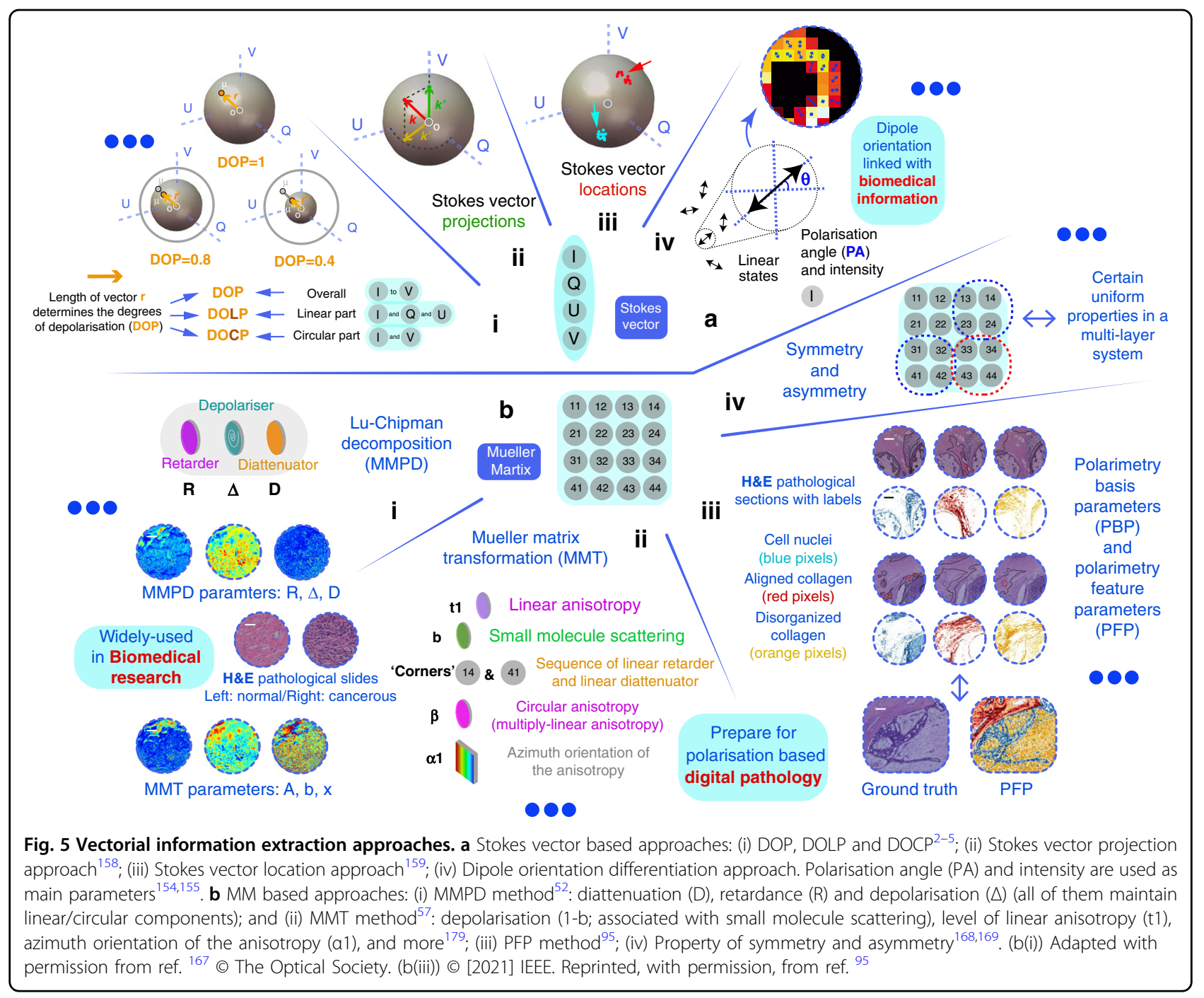

extract information that could be used in subsequent analysis $^{52-62}$.

\section{Information extraction from the vector properties of the light beam}

Several parameters can be calculated from the Stokes vector directly (see Fig. 5a (i) and previous section): such as the degree of polarisation (DOP), degree of linear polarisation (DOLP) and degree of circular polarisation (DOCP) of light. For a single uniform light beam, the DOP is 1 for fully polarised, 0 for unpolarised or completely depolarised, and between 0 and 1 for partially polarised. The DOP cannot be larger than 1. Despite containing four elements, a Stokes vector contains fewer than four degrees of freedom due to physical constraints. The Stokes vector can also be considered as an incoherent superposition of a completely polarised part and an unpolarised part ${ }^{3}$. Those parameters have been adopted in different polarimetric applications ${ }^{16,20,22,23,32,80,81}$. The polarisation angle (PA) and intensity of the linear SOP also can be defined, with respect to dipole orientation applications ${ }^{153-155}$ (see Fig. 5a (iv)). For a beam generated via an incoherent light source (such as a LED), the Stokes vectors can be directly added by scalar calculation. Therefore, partially polarised light can be divided into two parts-fully polarised/depolarised components ${ }^{3}$, i.e., $\mathbf{S}_{\text {total }}=\mathbf{S}_{\mathrm{u}}+\mathbf{S}_{\mathrm{p}}$; where $\mathbf{S}_{\mathrm{u}}$ and $\mathbf{S}_{\mathrm{p}}$ represent fully depolarised and polarised components, respectively.

For biomedical and clinical applications, characterising the vectorial properties of the outgoing light with a fixed incident SOP also showed great potential for structure identification $^{22-24,32}$. Wu \& Walsh reported that Stokes vector analysis with circular polarised illumination can reveal structural information about tissue ${ }^{156}$. Macdonald \& Meglinski showed that turbid tissue can be quantitatively analysed via Stokes vector measurement with an optical clearing technique ${ }^{157}$. Qi et al. proposed a method of Stokes vector analysis for ex vivo porcine tongue, stomach, kidney and other tissues based on circular polarised 
illumination ${ }^{158}$; the most useful information was provided by circular depolarisation and linear retardance, which can normally be provided via MM decomposition ${ }^{52,58}$ (as Stokes vector projections shown in Fig. 5a (ii)). Kunnen et al. employed Stokes vector detection with circular and elliptical incident SOPs for differentiation between healthy and cancerous lung tissues specifically using a Poincaré sphere illustration ${ }^{159}$ (as Stokes vector locations shown in Fig. 5a (iii)). Note that the circular SOP illumination is especially useful for biomedical analysis, as its effects are independent of the orientation of the anisotropic components that widely exist in biomedical specimens $^{20-24,32}$. What is more, its strong polarisation memory effect with respect to tissue-induced Mie-scattering has also gained attention ${ }^{160}$ (here the memory effect ${ }^{161}$ means that circular polarisation can survive many more scattering events than linear polarisation due to excessive forward scattering, hence it has higher probability to maintain the original information when passing through turbid tissue consisting of Mie-scattering particles that are comparable in size to the wavelength).

\section{Information extraction from the vector properties of the object}

Measurement of the full vector properties of biomedical targets requires illumination with multiple SOPs in combination with multiple analysing $\mathrm{SOPs}^{20-24}$. As we have mentioned above, the individual MM elements lack clear physical meanings, or explicit associations with microstructures $^{20-24}$. That is to say, vectorial characteristics of the object, like diattenuation, retardance, and depolarisation are encoded within the MM elements. For a complex optical system (like tissue), each MM element is always associated with more than one polarisation property. Hence, numerous MM decomposition methods were proposed to quantitatively characterise the optical and structural properties of the object ${ }^{52-59}$. One prevalent method is the Mueller matrix polar-decomposition (MMPD) proposed by Lu \& Chipman ${ }^{52}$, which has been used and validated in lots of applications for characterisation of biomedical or material samples ${ }^{58,162-167}$ (see Fig. 5b (i)); He et al. put forward the Mueller matrix transformation (MMT) with validations using phantom experiments and Monte Carlo simulations ${ }^{57}$. Based on the MMT concept, more rotation invariant parameters were extracted from the MM and applied to biomedical sample characterisation $^{167-169}$ (see Fig. 5b (ii)); Arteaga and colleagues derived Mueller matrix anisotropy coefficients (MMAC) to describe the level of different kinds of anisotropy for different polarisation systems ${ }^{56}$. Furthermore, other decomposition schemes were also developed, such as MM differential decomposition ${ }^{55}$, symmetric decomposition ${ }^{60,61}$ and Cloude decomposition ${ }^{62}$. Among the various decomposition approaches, different mathematical assumptions need to be made for different applications ${ }^{52-62}$, such as assuming a determined layer sequence of different fundamental polarisation components for a complex object, which in effect simplifies the matrix reciprocity problem ${ }^{52}$. Recently, those methods and related parameters have also been compared quantitatively with each other for the purpose of structural characterisation ${ }^{170-172}$. We can summarise the parameters derived via the above methods: MMPD: diattenuation (D), retardance (R) and depolarisation $(\Delta)$ (all of them maintain linear/circular components) ${ }^{52}$; MMT: depolarisation (1-b), level of linear anisotropy $\left(t_{1}\right)$, diattenuation property $\left(t_{2}\right)$, level of birefringence $\left(t_{3}\right)$ and fast axis orientation $\left(x_{3}\right)$ and more ${ }^{16,57}$; MMAC: horizontal linear anisotropy $(\alpha), 45^{\circ}$ linear anisotropy $(\beta)$ and circular anisotropy $(\gamma)$ respectively with respect to the global anisotropy of the $\mathrm{MM}^{56}$.

The MM contains fundamental physical characters like polarisance, diattenuation, retardance and depolarisation (shown in Fig. 2c); however, some concepts like anisotropy can be a combination of several fundamental polarisation processes ${ }^{16,57}$. It is worth mentioning that the depolarisation property-which is used for evaluating a SOP's disorder, randomness, or uncertainty ${ }^{3-5}-$ is also linked with the concept of entropy in polarimetric research $^{11,173}$. While the above parameters are derived from a full MM $(4 \times 4)$; Ghosh et al. and Wang et al. also reported works on $3 \times 3 \mathrm{MM}$ decomposition methods, related simulations and experiments, with an emphasis on biomedical applications ${ }^{174,175}$. In summary, the decomposed linear depolarisation and linear retardance from a $3 \times 3 \mathrm{MM}$ display similar qualitative relationships to the changes with respect to the microstructure of the sample, such as the density, molecule size, and orientation distributions of the scatterers as well as birefringence level of the interstitial medium ${ }^{174,175}$.

The MM decomposition methods all require different assumptions (strong or weak) such as matrix reciprocity, the order that polarisation effects happen in the media, or homogeneity for the tissue analysis ${ }^{52,58,176-178}$. Therefore, their decomposed values are not strictly physically determined, if the assumptions do not hold in reality, which may well be the case, as biological tissue has high spatial complexity ${ }^{58}$. However, extraction through the MM polarisation parameters that have less assumptions and clearer physical meaning is always something to strive for. Several works pointed in such a direction: (1) Gil et al. and $\mathrm{Li}$ et al. proposed different polarisation parameters with physical determination via the asymmetric properties of the MM elements ${ }^{168,169}$, by considering assumptions about layer constructions or the presence of absence of specific vector properties such as polarisance or diattenuation; (2) Dong et al. employed a data-driven machine learning technique to fit several polarimetry feature parameters (PFPs) for characterising determined 
pathological applications, such as detection of the abnormal areas of breast carcinoma and cervical cancerous tissue slices $^{95}$ (see Fig. 5b (iii)); 3) Breaking or restoring the symmetry (see Fig. 5b (iv)), based on analysis of different sub-regions of the MM, to extract determined information of the system is recently gaining interest ${ }^{179}$; The information extraction process is gradually developing from an analytical mathematics approach (equationbased, forward problem), to fitting or observing vectorial semantics/metrics (data-based, or shape/form-based inverse problem).

\section{Vectorial information analysis for biomedical applications}

Polarimetric techniques maintain unique advantages compared with other optical techniques: they can provide extra vectorial information through methods that are compatible with many existing optical systems, such as microscopes and endoscopes ${ }^{16,24,32,33,92,180}$. Much existing biomedical polarimetry research concerns sensing of bio-information in a label-free way without extraneous dyes ${ }^{16,22,24}$. In other areas, polarimetry can be used to characterise the vectorial information of fluorescence dyes, as the dipole orientation of the fluorophore is encoded in the polarisation state of the emitted light ${ }^{154,155}$. The SOP of such emission is always in a linear state; hence the polarisation angle (PA) and intensity of the linear SOP are quantities that can be harnessed, such as in biomedical applications in super-resolution microscopy $^{153,181,182}$. Here we briefly summarise common phantoms used for biomedical polarimetric techniques. These techniques include polarised wide-field microscopy ${ }^{16,24,183}$, polarised light spatial frequency imaging ${ }^{184}$, polarimetric endoscopy ${ }^{185-190}$, spectral light scattering polarimetry $18,82,191-193$, polarised fluorescence spectroscopy ${ }^{194-196}$, polarised confocal microscopy ${ }^{197}$, polarised Raman-spectroscopy ${ }^{198,199}$, polarised super-resolution microscopy ${ }^{154,155}$, polarisation sensitive optical coherence tomography ${ }^{200-218}$, non-diffraction beam polarimetry (such as Bessel beam based) ${ }^{219}$, polarisationresolved nonlinear microscopy (including second/third harmonic generation) ${ }^{220-226}$, and polarised speckle imaging $^{213,227}$ (several techniques will be mentioned again in the Discussion). The relationship between incoherence and depolarisation of the light should be kept in mind when considering coherence based polarimetric techniques: they are different but related optical concepts. If a polarised coherent beam passing through a scattering medium becomes incoherent, it can result in either polarised light or depolarised light. If after such a medium a polarised coherent beam changes into depolarised, the coherence property may still be maintained. For more details see ref. ${ }^{228}$. Several of the above techniques have also been adopted in three-dimensional (3D) imaging with signal integrations or sample segmentations ${ }^{229}$. However, numerous existing polarimetry techniques (within the scope of this review) fall into two-dimensional (2D) analysis $^{23-29}$. With the completion of the cutting-edge mathematical interpretations and methodologies (see "Discussion") there exists of course intriguing scope for further explorations.

In order to understand the interactions between polarised photons and biological specimens, and link the parameters obtained via the Stokes vector or MM with the biomedical microstructural information, a software phantom-Monte Carlo (MC) simulation-was proposed to give plausible explanations for the originality of the observed physical phenomena ${ }^{45,46}$. While biomedical samples are considered as turbid media with complex structures, different fundamental units to mimic the microstructural architecture have been employed: spherical scatterers ${ }^{35,46}$; cylindrical scatterers ${ }^{41,46}$; birefringent intermedia $^{37-39}$, multi-layered geometry ${ }^{45}$ and so on $^{46}$. MC simulations have successfully reproduced most of the important polarimetric characteristic features for biomedical samples ${ }^{16,230,231}$.

\section{Thin specimens}

Specimens and their mimicking phantoms can be thin or bulky, which also, in general, determines the configurations of the biomedical polarimetry. A transmissive geometry is used for the thin cases (see Fig. 6) which are less scattering, thus most of the incident photons would be transmitted. A backscattering geometry (see Fig. 7) is preferred for the bulk cases (ex vivo and in vivo) which are highly scattering and depolarising, thus most of the incident photons would be backscattered. There is no clear boundary between what constitutes thin or bulk tissues. Indeed, intermediate or mixed states can exist, for which both the transmission and backscattering photons can be detected simultaneously ${ }^{16,22,23,152}$. In response to the beginning of this section, biomedical polarimetry can be used in labelled or label-free measurement; Fig. 6 gives a summary for two types of the use of thin tissue polarimetry.

For label-based direction, polarimetry has found use in scientific applications, such as biomedical microscopy ${ }^{16}$. The vectorial information of the dipole emitters is encoded in the SOP of the detected light ${ }^{153,154}$. The dipole orientation (and the fluorescence intensity) polarimetric detection technique plays an important role in thin biomedical sample analysis: e.g., in fluorescence polarisation microscopy (FPM) ${ }^{194,195,232,233}$; FPM can be used to study the nuclear pore complex subcomplexes and the relative orientations $^{234}$, or be used to study different types of cytoskeleton such as actin, myosin, kinesin, microtubule and septin-those closely related with the performance of the dipole behaviours ${ }^{235-239}$-enabling research such as 


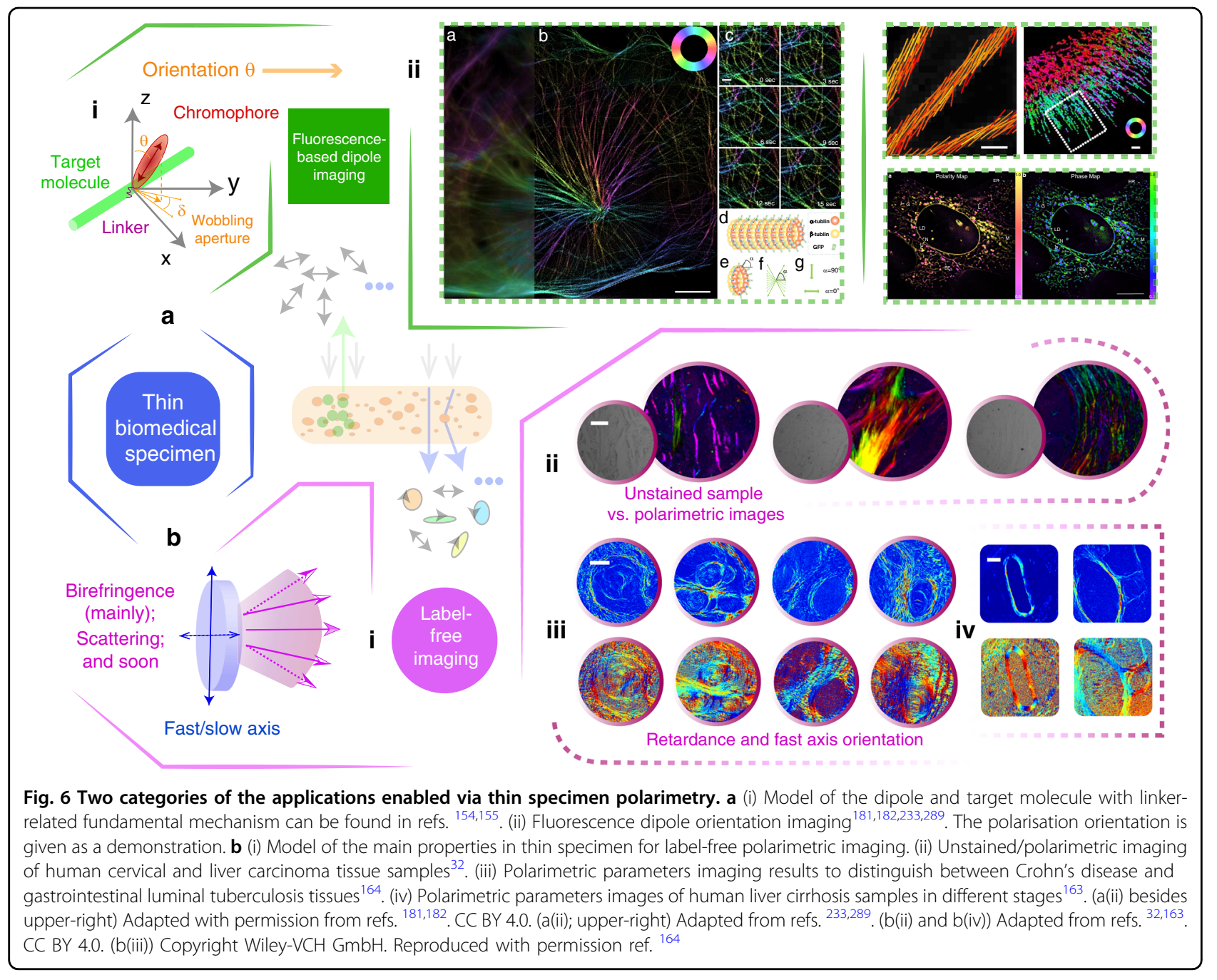

ATP and ADP binding ${ }^{237}$. Advanced research has been adopted in super-resolution imaging harnessing fluorescent dipoles via polarised illumination, with applications such as revealing heterogeneity and dynamics of subcellular lipid membranes ${ }^{181,240,241}$. These fluorescence anisotropy properties also belong to the fundamental polarisation properties that are encoded in the MM.

For label-free biomedical polarimetric research, especially in clinical/pathological related topics, cancerous tissues detection is an important application ${ }^{22-24}$. In the past decades, such polarimetric techniques have assisted the diagnosis of various cancerous tissues, such as human skin cancer $^{242}$, cervical cancer ${ }^{243-246}$, colon cancer $^{166,247-250}$, liver cancer ${ }^{163,251}$, breast cancer and gastrointestinal cancer $^{93-95,252}$. A typical bio-information analysis of polarimetric data is for quantitative evaluation of the fibrosis process among different stages of cancer development ${ }^{94,163}$. Beside the degree of fibrosis that can be quantified via biomedical polarimetry, the distribution of features in the fibrous regions also can serve as another characteristic parameter to assist the pathological diagnosis; this distribution can be readily extracted via polarisation information ${ }^{164,165,171}$. Intuitively, such structures contribute intrinsic birefringence mainly affecting the fourth row and fourth column of the target $\mathrm{MM}^{16}$. A good demonstration in ref. ${ }^{164}$ shows how polarimetric textural mapping of retardance properties can distinguish between Crohn's disease and gastrointestinal luminal tuberculosis tissues (see Fig. 6b). Some thin specimen phantoms, as found in ref. ${ }^{152}$, target the fundamental understanding of the constitution of certain biomedical specimens, such as using nanoparticles or microspheres. Moreover, polarimetry has recently been applied to other diseases detections including Alzheimer's disease and bladder outlet obstruction $^{24,253,254}$.

\section{Bulk specimens}

Polarisation techniques can help improve the image contrast of the superficial layers of tissues by eliminating multiply scattered photons from the deep layers ${ }^{20-24}$. The 


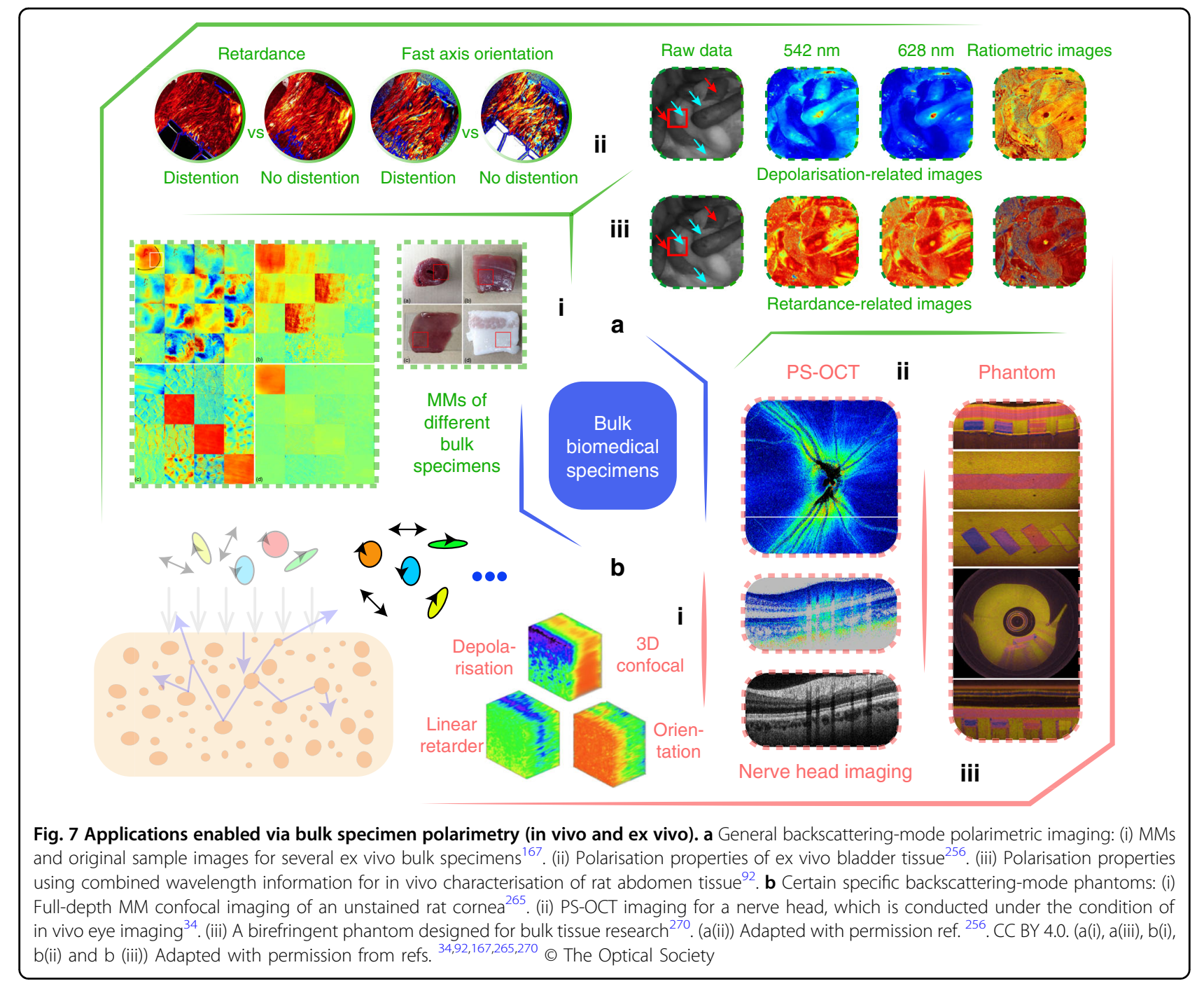

previous literature shows that more than $85 \%$ of cancers originate from the superficial epithelium, which means that polarisation imaging methods have great potential in screening and identifying cancer at an early stage ${ }^{255}$. This would be specifically useful for in vivo clinical diagnosis, such as for minimally invasive surgery $(\mathrm{MIS})^{24}$. Measurements in ex vivo thin tissue can use a transmissive geometry, whereas ex/in vivo bulk tissue detection would need backscattering configurations. Figure 7 gives a brief demonstration of certain current research topics related to bulk tissue polarimetry.

For polarimetric bulk tissue research, ex vivo detection plays an important role ${ }^{22-24}$. For example, collagen fibres, which widely exist in tissues and organs such as tendons, skin and bladder (from porcine, swine, lobster, calf or other animals ${ }^{156,256-259}$ ), skeletal and myocardial muscle fibres $^{260-262}$, and elastin fibres are widely used due to their linear birefringence properties ${ }^{20-23,164}$. The alignment directions of all such fibrous structures are also linked with the fast axis orientation of the generated linear birefringence $^{16,165,171}$. Furthermore, the scattering of bulk media is also studied via the extracted depolarisation ${ }^{16,167,263}$. The retardance and depolarisation related properties are the dominant parts of the vectorial properties of bulk tissues, as the magnitude of diattenuation for majority of tissue is typically very small ${ }^{158}$, with several exceptions like skeletal and myocardial muscles ${ }^{167}$ (see Fig. 7a (i)). Previous research analysing muscle tissue ${ }^{264}$ showed lower retardance compared with tendon tissue, owing to the cellularity of these tissues. Sections of the bulk myocardial fibre tissues showed two circularly aligned ring-shaped fibrous structures (see Fig. 7a (i)), revealing their anisotropic properties ${ }^{167}$. The different anisotropic vectorial information obtained from polarimetric measurements can be very helpful for the discrimination and identification of different fibrous structures in tissues ${ }^{164,165,167}$.

While ex vivo studies are mainly oriented towards fundamental research ${ }^{24,152,171}$ (e.g., understanding the vectorial 
properties characterisation; see Fig. 7a (i), (ii), b (i)), in vivo bulk tissue polarimetry is geared towards applications $^{24,256,265}$. Typical backscattering-mode polarimetry includes polarisation endoscopy ${ }^{24}$, reflection MM microscopy $^{16,266}$, MM colposcopy ${ }^{180,267}$, wide-field handheld polarimetry ${ }^{16}$, and PS-OCT ${ }^{268}$ (see Fig. 7a (iii), b (ii)), targeted to clinical diagnosis in vivo. As a promising in vivo, label-free diagnostic tool, polarisation endoscopic imaging has been implemented inside rat abdomen, revealing the small bowel, stomach, liver and fat with different polarisation characters ${ }^{92}$. Recent work also includes development of several different types of MM endoscope ${ }^{188-190,256}$, and extension into the spectral domain (with certain fixed wavelengths) $^{92}$ (see Fig. 7a (iii)). PS-OCT ${ }^{200-218}$ is specifically used for in vivo ophthalmic imaging, where polarimetric data accompanied with clinical analysis has been demonstrated, for retinal imaging ${ }^{268}$ (see Fig. 7b (ii)). Other types of bulk tissue analysis, such as human lung cancerous tissue $^{159}$ and skin tissue ${ }^{269}$, show good prospects for future clinical diagnosis ${ }^{16,22-24}$.

While thin samples can feature multiple-scattering process, such processes are of course more significant in bulk samples $^{20-22}$. Considering the intriguing scope of the polarimetric technique for in vivo clinical diagnosis, beside the simulations, there is a need for complex phantoms, such as those exhibiting birefringence (see Fig. 7b (iii)) or depolarisation, to establish reliable processes for investigation of complex scattering mechanisms ${ }^{270,271}$. A recent review has summarised various phantoms for both thin and bulk samples ${ }^{152}$. Microspheres, silicon-based phantoms,

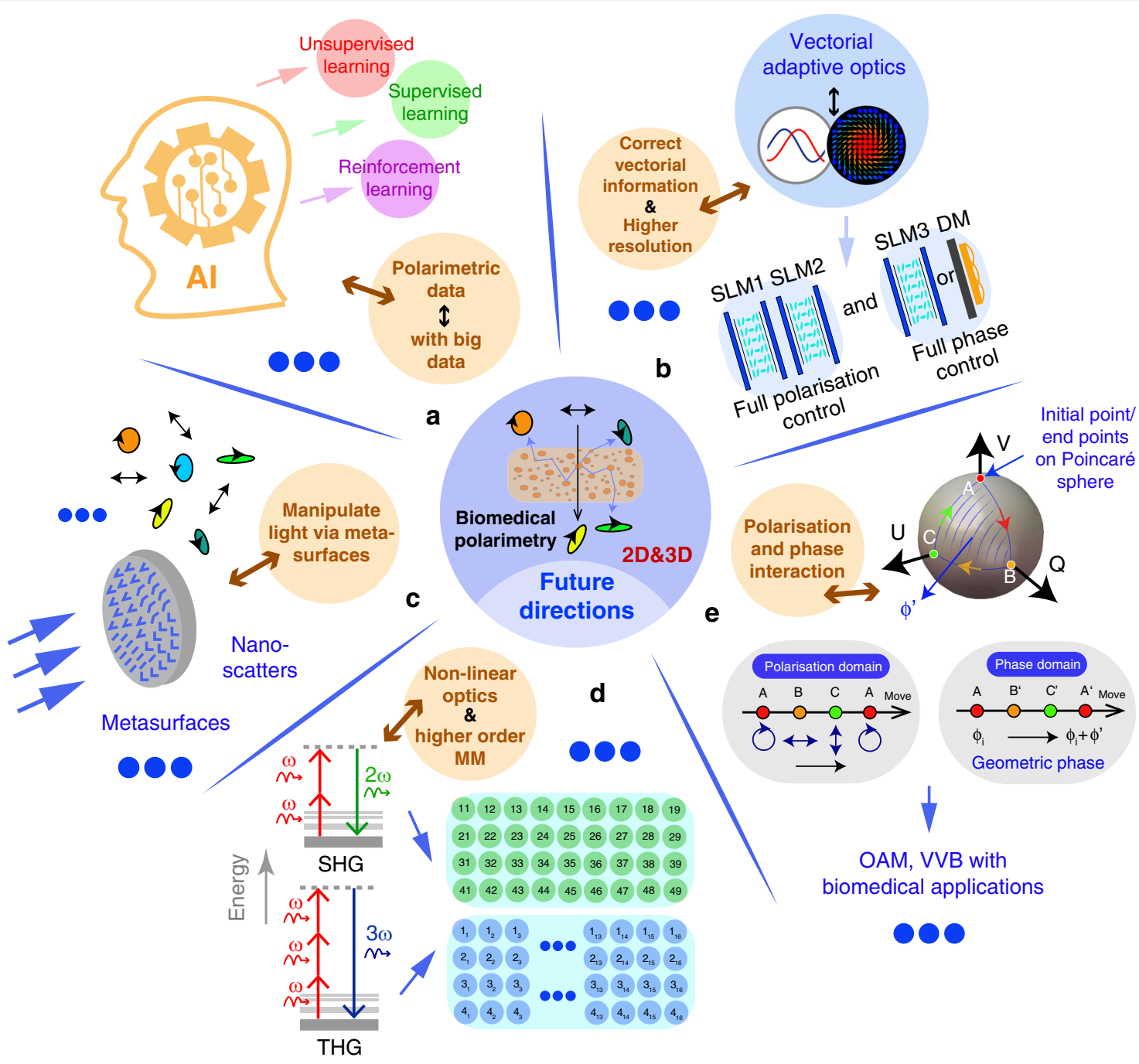

Fig. 8 Future directions for biomedical polarimetry. a Combination with machine learning techniques and big data. b Combination with vectorial adaptive optics. c Combination with metasurface based techniques. $\mathbf{d}$ Combination with nonlinear techniques, such as SHG and THG, in which a high order MM is required. e Combination with absolute phase information, such as geometric phase, with potential applications related with orbital angular momentum (OAM) and vector vortex beam (WB) manipulations 
nanoparticles, cylindrical scatterers, and birefringent/ dichroism films ${ }^{35,158,270,272}$ have all been employed in various validations. In vivo biomedical polarimetry and its related applications clearly offer a large space for future exploration.

\section{Directions for advanced biomedical polarimetry and future prospects}

Biomedical applications of polarimetry have attracted substantial attention. We hope this short review paper gives readers a general overview from fundamental polarisation concepts, through polarimetric techniques, to recent biomedical and clinical applications ${ }^{7,16,20-24,29,34,63,79,152}$. In addition to the summaries of recent research trends explained above, we provide here some further perspective on prospects in this application area, considering the use of polarimetry in a multimodal combination with other advanced technologies (see Fig. 8 for a summary).

Firstly, the fast development of machine learning (ML) is clearly going to have an impact on this field ${ }^{95,273,274}$. Such data-driven techniques may pave new directions for biomedical polarimetry, either through improving the quality of polarimetry (such as overcoming the numerous sources of error) or through enhanced information extraction $^{68,95}$. One possibility is to use low-resolution information to reconstruct high-resolution patterns (following the spirit of works such as refs. ${ }^{275,276}$ ). Secondly, while ML is geared towards improving the information processing aspects of polarimetry, new adaptive optics techniques can be used to extend the capabilities of polarimetry through full vectorial beam control. This could enable enhanced polarisation imaging resolution physically via beam shaping and compensation of polarisation errors ${ }^{277-280}$. Thirdly, the emerging techniques based on metasurfaces-subwavelength arrays of nanoscatterers that can modify polarisation-have been adopted for polarimetry ${ }^{281}$, as well as for 3D polarisation control $^{282}$. Such developments may bring new opportunities for advanced biomedical polarimetry, such as forming compact vectorial sensors ${ }^{24,283}$ for deep tissue information extraction. Fourthly, second harmonic generation (SHG) and third harmonic generation (THG) based 3D MM techniques have been proposed ${ }^{284-287}$. These are described by extended MMs that are more complicated than $4 \times 4$ MMs used for linear scattering $(4 \times 9$ and $4 \times 16$ elements, respectively, for SHG and THG ${ }^{284,285}$. For these methods, further advanced information extraction and analysis approaches are of course intriguing. Finally, the intensity and wavelength have been utilised together with polarisation in polarimetry for a long time. However, the absolute phase informationespecially geometric phase-related techniques ${ }^{8,10}$-may again open windows for new biomedical polarimetry approaches with multi-modal performance.

\section{Acknowledgements}

The project was supported by the European Research Council (AdOMiS, no. 695140); Shenzhen Fundamental Research and Discipline Layout Project (JCYJ20170412170814624); The first author C.H. (also the figure maker) would like to thank Prof. Jessica C Ramella-Roman at Florida International University, Prof. Daniel S Elson at Imperial College London, Prof. Christoph K Hitzenberger at Medical University of Vienna, Prof. Johannes Fitzgerald de Boer at Vrije Universiteit Amsterdam, Prof. Yoshiaki Yasuno at University of Tsukuba, Prof. Martin Villiger at Harvard Medical School, Prof. Nan Zeng and Prof. Yonghong He at Tsinghua University, Dr. Long Chen at University of Oxford for useful discussions and/or support through the provision of the original graphics of their works.

\section{Author details}

'Department of Engineering Science, University of Oxford, Parks Road, Oxford OX1 3PJ, UK. ${ }^{2}$ Guangdong Engineering Center of Polarisation Imaging and Sensing Technology, Tsinghua Shenzhen International Graduate School, Tsinghua University, 518055 Shenzhen, China. Institute of Biopharmaceutical and Health Engineering, Tsinghua Shenzhen International Graduate School, Tsinghua University, 518055 Shenzhen, China. ${ }^{4}$ Department of Physics, Tsinghua University, 100084 Beijing, China. ${ }^{5}$ Department of Biomedical Engineering, Tsinghua University, 100084 Beijing, China

\section{Conflict of interest}

The authors declare no competing interests.

Received: 26 April 2021 Revised: 30 August 2021 Accepted: 1 September 2021

Published online: 22 September 2021

\section{References}

1. Ronchi, V. \& Barocas, V. The Nature of Light: An Historical Survey (Hanvard University Press, 1970).

2. Huard, S. Polarization of Light (Wiley, 1997).

3. Goldstein, D. Polarized Light 2nd edn (Marcel Dekker, 2003).

4. Chipman, R. A., Lam, W. S. T. \& Young, G. Polarized Light and Optical Systems (CRC Press, 2018).

5. Pérez, J. J. G. \& Ossikovski, R. Polarized Light and the Mueller Matrix Approach (CRC Press, 2017).

6. Zhan, Q. W. Cylindrical vector beams: from mathematical concepts to applications. Adv. Opt. Photonics 1, 1-57 (2009).

7. Rosales-Guzmán, C., Ndagano, B. \& Forbes, A. A review of complex vector light fields and their applications. J. Opt. 20, 123001 (2018).

8. Forbes, A., De Oliveira, M. \& Dennis, M. R. Structured light. Nat. Photonics $\mathbf{1 5}$ 253-262 (2021).

9. Wang, J. W., Castellucci, F. \& Franke-Arnold, S. Vectorial light-matter interaction: exploring spatially structured complex light fields. AVS Quantum Sci. 2, $031702(2020)$

10. Slussarenko, S. et al. Guiding light via geometric phases. Nat. Photonics $\mathbf{1 0}$ 571-575 (2016).

11. Cloude, S. Polarisation: Applications in Remote Sensing (OUP Oxford, 2009).

12. Ndagano, B. et al. Characterizing quantum channels with non-separable states of classical light. Nat. Phys. 13, 397-402 (2017).

13. Marrucci, L., Manzo, C. \& Paparo, D. Optical spin-to-orbital angular momentum conversion in inhomogeneous anisotropic media. Phys. Rev. Lett. 96 163905 (2006)

14. Bliokh, K. Y. et al. Spin-orbit interactions of light. Nat. Photonics $\mathbf{9}, 796-808$ (2015).

15. Schulz, M. et al. Giant intrinsic circular dichroism of prolinol-derived squaraine thin films. Nat. Commun. 9, 2413 (2018).

16. He, H. H. et al. Mueller matrix polarimetry-an emerging new tool for characterizing the microstructural feature of complex biological specimen. $J$. Lightwave Technol. 37, 2534-2548 (2019).

17. Oldenbourg, R. A new view on polarization microscopy. Nature $\mathbf{3 8 1}, 811-812$ (1996).

18. Gurjar, R. S. et al. Imaging human epithelial properties with polarized lightscattering spectroscopy. Nat. Med. 7, 1245-1248 (2001).

19. Qiu, L. et al. Multispectral scanning during endoscopy guides biopsy of dysplasia in Barrett's esophagus. Nat. Med. 16, 603-606 (2010). 
20. Ghosh, N. \& Vitkin, I. A. Tissue polarimetry: concepts, challenges, applications, and outlook. J. Biomed. Opt. 16, 110801 (2011).

21. Novikova, T. et al. Special section guest editorial: polarized light for biomedical applications. J. Biomed. Opt. 21, 071001 (2016)

22. Tuchin, V. V. Polarized light interaction with tissues. J. Biomed. Opt. 21, 071114 (2016).

23. Ramella-Roman, J. C., Saytashev, I. \& Piccini, M. A review of polarization-based imaging technologies for clinical and preclinical applications. J. Opt. 22, 123001 (2020).

24. Qi, J. \& Elson, D. S. Mueller polarimetric imaging for surgical and diagnostic applications: a review. J. Biophotonics 10, 950-982 (2017).

25. Fujiwara, H. Spectroscopic Ellipsometry: Principles and Applications (John Wiley \& Sons, 2007)

26. Azzam, R. M. A., Bashara, N. M. \& Ballard, S. S. Ellipsometry and polarized light. Phys. Today 31, 72 (1978)

27. Mendoza-Galván, A. et al. Mueller matrix spectroscopic ellipsometry study of chiral nanocrystalline cellulose films. J. Opt. 20, 024001 (2018).

28. Azzam, R. M. A. Stokes-vector and Mueller-matrix polarimetry. J. Optical Soc. Am. A 33, 1396-1408 (2016).

29. Azzam, R. M. A. Mueller-matrix ellipsometry: a review. Proc. SPIE 3121 369-405 (1997).

30. Song, B. K. et al. Broadband optical properties of graphene and HOPG investigated by spectroscopic Mueller matrix ellipsometry. Appl. Surf. Sci. 439, 1079-1087 (2018).

31. Jiang, $\mathrm{H}$. et al. Characterization of volume gratings based on distributed dielectric constant model using Mueller matrix ellipsometry. Appl. Sci. 9, 698 (2019).

32. Chang, J. T. et al. Division of focal plane polarimeter-based $3 \times 4$ Mueller matrix microscope: a potential tool for quick diagnosis of human carcinoma tissues. J. Biomed. Opt. 21, 056002 (2016).

33. Fu, Y. F. et al. Flexible $3 \times 3$ Mueller matrix endoscope prototype for cancer detection. IEEE Trans. Instrum. Meas. 67, 1700-1712 (2018).

34. De Boer, J. F., Hitzenberger, C. K. \& Yasuno, Y. Polarization sensitive optical coherence tomography - a review. Biomed. Opt. Express 8, 1838-1873 (2017).

35. Ghosh, N., Patel, H. S. \& Gupta, P. K. Depolarization of light in tissue phantoms -effect of a distribution in the size of scatterers. Opt. Express 11, 2198-2205 (2003).

36. Kienle, A. \& Hibst, R. Light guiding in biological tissue due to scattering. Phys. Rev. Lett. 97, 018104 (2006).

37. Baravian, C., Dillet, J. \& Decruppe, J. P. Birefringence determination in turbid media. Phys. Rev. E 75, 032501 (2007).

38. Wang, X. D. \& Wang, L. V. Propagation of polarized light in birefringent turbid media: a Monte Carlo study. J. Biomed. Opt. 7, 279-290 (2002).

39. Wang, X. D. \& Wang, L. V. Propagation of polarized light in birefringent turbid media: time-resolved simulations. Opt. Express 9, 254-259 (2001).

40. Du, E. et al. Two-dimensional backscattering Mueller matrix of spherecylinder birefringence media. J. Biomed. Opt. 17, 126016 (2012).

41. He, H. H. et al. Application of sphere-cylinder scattering model to skeletal muscle. Opt. Express 18, 15104-15112 (2010).

42. Chen, D. S. et al. Mueller matrix polarimetry for characterizing microstructural variation of nude mouse skin during tissue optical clearing. Biomed. Opt. Express 8, 3559-3570 (2017).

43. Donner, C. \& Jensen, H. W. Light diffusion in multi-layered translucent materials. ACM Trans. Graph. 24, 1032-1039 (2005).

44. Van De Hulst, H. C. Light Scattering by Small Particles (Dover Publications, 1981).

45. Wang, L. H., Jacques, S. L. \& Zheng, L. Q. MCML-Monte Carlo modeling of light transport in multi-layered tissues. Comput. Methods Prog. Biomed. 47, 131-146 (1995).

46. Yun, T. L. et al. Monte Carlo simulation of polarized photon scattering in anisotropic media. Opt. Express 17, 16590-16602 (2009).

47. Brosseau, C. Fundamentals of Polarized Light: a Statistical Optics Approach (Wiley-Interscience, 1998).

48. Born, M. \& Wolf, E. Principles of Optics: Electromagnetic Theory of Propagation, Interference and Diffraction of Light (Elsevier, 2013).

49. Ling, X. H. et al. Recent advances in the spin Hall effect of light. Rep. Prog. Phys. 80, 066401 (2017).

50. Rubinsztein-Dunlop, H. et al. Roadmap on structured light. J. Opt. 19, 013001 (2017)

51. Bass, M. et al. Handbook of Optics, Volume N: Optical Properties of Materials 3rd edn. (McGraw-Hill Education, 2009).
52. Lu, S. Y. \& Chipman, R. A. Interpretation of Mueller matrices based on polar decomposition. J. Optical Soc. Am. A 13, 1106-1113 (1996).

53. Arteaga, O. \& Canillas, A. Pseudopolar decomposition of the Jones and Mueller-Jones exponential polarization matrices. J. Optical Soc. Am. A 26, 783-793 (2009).

54. Ossikovski, R., De Martino, A. \& Guyot, S. Forward and reverse product decompositions of depolarizing Mueller matrices. Opt. Lett. 32, 689-691 (2007).

55. Ortega-Quijano, N. \& Arce-Diego, J. L. Mueller matrix differential decomposition. Opt. Lett. 36, 1942-1944 (2011).

56. Arteaga, O., Garcia-Caurel, E. \& Ossikovski, R. Anisotropy coefficients of a Mueller matrix. J. Optical Soc. Am. A 28, 548-553 (2011).

57. He, H. H. et al. A possible quantitative Mueller matrix transformation technique for anisotropic scattering media/Eine mögliche quantitative MüllerMatrix-Transformations-Technik für anisotrope streuende Medien. Photonics Lasers Med. 2, 129-137 (2013).

58. Ghosh, N., Wood, M. F. G. \& Vitkin, I. A. Mueller matrix decomposition for extraction of individual polarization parameters from complex turbid media exhibiting multiple scattering, optical activity, and linear birefringence. $J$. Biomed. Opt. 13, 044036 (2008).

59. Gil, J. J. Characteristic properties of Mueller matrices. J. Optical Soc. Am. A 17, 328-334 (2000).

60. Ossikovski, R. Analysis of depolarizing Mueller matrices through a symmetric decomposition. J. Optical Soc. Am. A 26, 1109-1118 (2009).

61. Vizet, J. \& Ossikovski, R. Symmetric decomposition of experimental depolarizing Mueller matrices in the degenerate case. Appl. Opt. 57, 1159-1167 (2018).

62. Cloude, S. R. Group theory and polarisation algebra. Optik 75, $26-36$ (1985).

63. Pezzaniti, J. L. \& Chipman, R. A. Mueller matrix imaging polarimetry. Optical Eng. 34, 1558-1568 (1995).

64. Azzam, R. M. A. Photopolarimetric measurement of the Mueller matrix by Fourier analysis of a single detected signal. Opt. Lett. 2, 148-150 (1978).

65. Goldstein, D. H. Mueller matrix dual-rotating retarder polarimeter. Appl. Opt. 31, 6676-6683 (1992).

66. Smith, M. H. Optimization of a dual-rotating-retarder Mueller matrix polarimeter. Appl. Opt. 41, 2488-2493 (2002).

67. Dubreuil, M. et al. Snapshot Mueller matrix polarimeter by wavelength polarization coding. Opt. Express 15, 13660-13668 (2007).

68. He, C. et al. Full Poincare mapping for ultra-sensitive polarimetry. Preprint at https://arxiv.org/abs/2101.09372 (2021).

69. Sabatke, D. S. et al. Optimization of retardance for a complete Stokes polarimeter. Opt. Lett. 25, 802-804 (2000).

70. Tyo, J. S. Design of optimal polarimeters: maximization of signal-to-noise ratio and minimization of systematic error. Appl. Opt. 41, 619-630 (2002)

71. He, C. et al. Linear polarization optimized Stokes polarimeter based on fourquadrant detector. Appl. Opt. 54, 4458-4463 (2015).

72. He, C. et al. Complex vectorial optics through gradient index lens cascades. Nat. Commun. 10, 4264 (2019).

73. Li, X. B. et al. Learning-based denoising for polarimetric images. Opt. Express 28, 16309-16321 (2020).

74. Abubakar, A. et al. A hybrid denoising algorithm of BM3D and KSVD for Gaussian noise in DoFP polarization images. IEEE Access 8, 57451-57459 (2020).

75. Bueno, J. M. Polarimetry using liquid-crystal variable retarders: theory and calibration. J. Opt. A: Pure Appl. Opt. 2, 216-222 (2000).

76. Skumanich, A. et al. The calibration of the advanced Stokes polarimeter. Astrophys. J. Suppl. Ser. 110, 357-380 (1997).

77. Arteaga, O. et al. Mueller matrix polarimetry with four photoelastic modulators: theory and calibration. Appl. Opt. 51, 6805-6817 (2012).

78. Smith, M. H. et al. Infrared Stokes polarimeter calibration. Proc. SPIE $\mathbf{4 1 3 3}$ $55-64$ (2000).

79. Tyo, J. S. et al. Review of passive imaging polarimetry for remote sensing applications. Appl. Opt. 45, 5453-5469 (2006).

80. Jacques, S. L., Ramella-Roman, J. C. \& Lee, K. Imaging skin pathology with polarized light. J. Biomed. Opt. 7, 329-340 (2002).

81. Jacques, S. L., Roman, J. R. \& Lee, K. Imaging superficial tissues with polarized light. Lasers Surg. Med. 26, 119-129 (2000).

82. Demos, S. G., Radousky, H. B. \& Alfano, R. R. Deep subsurface imaging in tissues using spectral and polarization filtering. Opt. Express 7, 23-28 (2000).

83. Demos, S. G. \& Alfano, R. R. Optical polarization imaging. Appl. Opt. 36, 150-155 (1997). 
84. Groner, W. et al. Orthogonal polarization spectral imaging: a new method for study of the microcirculation. Nat. Med. 5, 1209-1212 (1999).

85. Bargo, P. R. \& Kollias, N. Measurement of skin texture through polarization imaging. Br. J. Dermatol. 162, 724-731 (2010).

86. Sridhar, S. \& Da Silva, A. Enhanced contrast and depth resolution in polarization imaging using elliptically polarized light. J. Biomed. Opt. 21, 071107 (2016).

87. Collett, E. Measurement of the four Stokes polarization parameters with a single circular polarizer. Opt. Commun. 52, 77-80 (1984).

88. Laude-Boulesteix, B. et al. Mueller polarimetric imaging system with liquid crystals. Appl. Opt. 43, 2824-2832 (2004).

89. Sornsin, E. A. \& Chipman, R. A. Mueller matrix polarimetry of electro-optic PLZT spatial light modulators. Proc. SPIE 2873, 196-201 (1996).

90. Peinado, A., Lizana, A. \& Campos, J. Optimization and tolerance analysis of a polarimeter with ferroelectric liquid crystals. Appl. Opt. 52, 5748-5757 (2013).

91. Alali, S., Gribble, A. \& Vitkin, I. A. Rapid wide-field Mueller matrix polarimetry imaging based on four photoelastic modulators with no moving parts. Opt. Lett. 41, 1038-1041 (2016).

92. Qi, J. et al. Narrow band $3 \times 3$ Mueller polarimetric endoscopy. Biomed. Opt. Express 4, 2433-2449 (2013).

93. Dong, $Y$. et al. Probing variations of fibrous structures during the development of breast ductal carcinoma tissues via Mueller matrix imaging. Biomed. Opt. Express 11, 4960-4975 (2020).

94. Dong, Y. et al. Quantitatively characterizing the microstructural features of breast ductal carcinoma tissues in different progression stages by Mueller matrix microscope. Biomed. Opt. Express 8, 3643-3655 (2017).

95. Dong, Y. et al. Deriving polarimetry feature parameters to characterize microstructural features in histological sections of breast tissues. IEEE Trans. Biomed. Eng. 68, 881-892 (2021).

96. Hagen, N., Oka, K. \& Dereniak, E. L. Snapshot Mueller matrix spectropolarimeter. Opt. Lett. 32, 2100-2102 (2007).

97. Azzam, R. M. A. Division-of-amplitude photopolarimeter (DOAP) for the simultaneous measurement of all four Stokes parameters of light. Opt. Acta.: Int. J. Opt. 29, 685-689 (1982).

98. Jellison, G. E. Jr. Four-channel polarimeter for time-resolved ellipsometry. Opt. Lett. 12, 766-768 (1987).

99. Compain, E. \& Drevillon, B. Broadband division-of-amplitude polarimeter based on uncoated prisms. Appl. Opt. 37, 5938-5944 (1998).

100. Peinado, A. et al. Conical refraction as a tool for polarization metrology. Opt. Lett. 38, 4100-4103 (2013).

101. Haigh, J. A., Kinebas, Y. \& Ramsay, A. J. Inverse conoscopy: a method to measure polarization using patterns generated by a single birefringent crystal. Appl. Opt. 53, 184-188 (2014).

102. Chang, J. T. et al. Single-shot spatially modulated Stokes polarimeter based on a GRIN lens. Opt. Lett. 39, 2656-2659 (2014).

103. Bhandari, P., Voss, K. J. \& Logan, L. An instrument to measure the downwelling polarized radiance distribution in the ocean. Opt. Express 19, 17609-17620 (2011).

104. Pezzaniti, J. L. \& Chenault, D. B. A division of aperture MWIR imaging polarimeter. Proc. SPIE 5888, 58880V (2005).

105. Zimmerman, B. G. et al. Pinhole array implementation of star test polarimetry. Proc. SPIE 8949, 894912 (2014).

106. Chun, C. S. L., Fleming, D. L. \& Torok, E. J. Polarization-sensitive thermal imaging. Proc. SPIE 2234, 275-286 (1994).

107. Nordin, G. P. et al. Micropolarizer array for infrared imaging polarimetry. J. Optical Soc. Am. A 16, 1168-1174 (1999).

108. Andreou, A. G. \& Kalayjian, Z. K. Polarization imaging: principles and integrated polarimeters. IEEE Sens. J. 2, 566-576 (2002).

109. Chen, Z. Y., Wang, X. \& Liang, R. G. Calibration method of microgrid polarimeters with image interpolation. Appl. Opt. 54, 995-1001 (2015).

110. Gao, S. K. \& Gruev, V. Bilinear and bicubic interpolation methods for division of focal plane polarimeters. Opt. Express 19, 26161-26173 (2011).

111. Gao, S. K. \& Gruev, V. Gradient-based interpolation method for division-offocal-plane polarimeters. Opt. Express 21, 1137-1151 (2013).

112. Gruev, V., Perkins, R. \& York, T. CCD polarization imaging sensor with aluminum nanowire optical filters. Opt. Express 18, 19087-19094 (2010).

113. Hsu, W. L. et al. Polarization microscope using a near infrared full-Stokes imaging polarimeter. Opt. Express 23, 4357-4368 (2015).

114. Liu, Y. et al. Complementary fluorescence-polarization microscopy using division-of-focal-plane polarization imaging sensor. J. Biomed. Opt. 17, 116001 (2012).
115. Millerd, J. et al. Pixelated phase-mask dynamic interferometers. In Fringe 2005 (ed Osten, W.) 640-647 (Springer, 2006).

116. Ratliff, B. M., LaCasse, C. F. \& Tyo, J. S. Interpolation strategies for reducing IFOV artifacts in microgrid polarimeter imagery. Opt. Express 17, 9112-9125 (2009).

117. Ratliff, B. M. et al. Dead pixel replacement in LWIR microgrid polarimeters. Opt. Express 15, 7596-7609 (2007).

118. Tyo, J. S., LaCasse, C. F. \& Ratliff, B. M. Total elimination of sampling errors in polarization imagery obtained with integrated microgrid polarimeters. Opt. Lett. 34, 3187-3189 (2009).

119. York, T. \& Gruev, V. Calibration method for division of focal plane polarimeters in the optical and near-infrared regime. Proc. SPIE 8012, 80120H (2011).

120. York, T. et al. Bioinspired polarization imaging sensors: from circuits and optics to signal processing algorithms and biomedical applications. Proc. IEEE 102, 1450-1469 (2014)

121. Zhang, Z. G. et al. Nano-fabricated pixelated micropolarizer array for visible imaging polarimetry. Rev. Sci. Instrum. 85, 105002 (2014).

122. Zhao, X. J. et al. Patterned dual-layer achromatic micro-quarter-wave-retarder array for active polarization imaging. Opt. Express 22, 8024-8034 (2014)

123. Oka, K. \& Saito, N. Snapshot complete imaging polarimeter using Savart plates. Proc. SPIE 6295, 629508 (2006)

124. Suárez-Bermejo, J. C. et al. Mueller matrix polarimetry using full Poincaré beams. Opt. Lasers Eng. 122, 134-141 (2019).

125. Goldstein, D. H. \& Chipman, R. A. Error analysis of a Mueller matrix polarimeter. J. Opt. Soc. Am. A 7, 693-700 (1990).

126. Ahmad, J. E. \& Takakura, Y. Error analysis for rotating active Stokes-Mueller imaging polarimeters. Opt. Lett. 31, 2858-2860 (2006).

127. Dai, H. \& Yan, C. X. Measurement errors resulted from misalignment errors of the retarder in a rotating-retarder complete Stokes polarimeter. Opt. Express 22, 11869-11883 (2014).

128. Mu, T. K. et al. Error analysis of single-snapshot full-Stokes division-of-aperture imaging polarimeters. Opt. Express 23, 10822-10835 (2015).

129. Macias-Romero, C. \& Török, P. Eigenvalue calibration methods for polarimetry J. Eur. Opt. Soc. Rapid Publ. 7, 12004 (2012)

130. Compain, E., Poirier, S. \& Drevillon, B. General and self-consistent method for the calibration of polarization modulators, polarimeters, and Mueller-matrix ellipsometers. Appl. Opt. 38, 3490-3502 (1999).

131. De Martino, A. et al. General methods for optimized design and calibration of Mueller polarimeters. Thin Solid Films 455-456, 112-119 (2004).

132. Marenko, V. \& Molebnaya, T. Optimization of stokes polarimeters employing a measurement of 4intensities. Sov. J. Opt. Technol. 57, 452-455 (1990).

133. Ambirajan, A. \& Look, D. C. Jr. Optimum angles for a Mueller matrix polarimeter. Proc. SPIE 2265, 314-326 (1994).

134. Ambirajan, A. \& Look, D. C. Jr. Optimum angles for a polarimeter: part I. Opt. Eng. 34, 1651-1655 (1995).

135. Tyo, J. S. Optimum linear combination strategy for an $\mathrm{N}$-channel polarization-sensitive imaging or vision system. J. Opt. Soc. Am. A 15, 359-366 (1998).

136. Tyo, J. S. Relation between system optimization and systematic errors in Stokes vector polarimeters. Proc. SPIE 4481, 22-30 (2002).

137. Tyo, J. S. Noise equalization in Stokes parameter images obtained by use of variable-retardance polarimeters. Opt. Lett. 25, 1198-1200 (2000).

138. Azzam, R. M. A., Elminyawi, I. M. \& El-Saba, A. M. General analysis and optimization of the four-detector photopolarimeter. J. Opt. Soc. Am. A 5, 681-689 (1988).

139. Tyo, J. S. Considerations in polarimeter design. Proc. SPIE 4133, 65-74 (2000).

140. Peinado, A. et al. Optimization and performance criteria of a Stokes polarimeter based on two variable retarders. Opt. Express 18, 9815-9830 (2010).

141. Foreman, M. R., Favaro, A. \& Aiello, A. Optimal frames for polarization state reconstruction. Phys. Rev. Lett. 115, 263901 (2015).

142. Foreman, M. R. \& Goudail, F. On the equivalence of optimization metrics in Stokes polarimetry. Opt. Eng. 58, 082410 (2019).

143. Twietmeyer, K. M. \& Chipman, R. A. Optimization of Mueller matrix polarimeters in the presence of error sources. Opt. Express 16, 11589-11603 (2008).

144. Beckley, A. M., Brown, T. G. \& Alonso, M. A. Full Poincaré beams. Opt. Express 18, 10777-10785 (2010).

145. Beckley, A. M. Polarimetry and Beam Apodization Using Stress-engineered Optical Elements. PhD thesis (University of Rochester, New York, 2012).

146. Dewage, A. A. G. \& Brown, T. Interferometric polarimetry using full-Poincar, beams. Proc. SPIE 11701, 117010N (2021).

147. Vella, A. \& Alonso, M. A. Optimal birefringence distributions for imaging polarimetry. Opt. Express 27, 36799-36814 (2019). 
148. Vella, A. \& Alonso, M. A. Chapter Seven-maximum likelihood estimation in the context of an optical measurement. Prog. Opt. 65, 231-311 (2020).

149. Vella, A. J. Description and Applications of Space-variant Polarization States and Elements. PhD thesis. (University of Rochester, New York, 2018).

150. Ramkhalawon, R. D., Brown, T. G. \& Alonso, M. A. Imaging the polarization of a light field. Opt. Express 21, 4106-4115 (2013).

151. Zimmerman, B. G. \& Brown, T. G. Star test image-sampling polarimeter. Opt. Express 24, 23154-23161 (2016)

152. Chue-Sang, J. et al. Optical phantoms for biomedical polarimetry: a review. J. Biomed. Opt. 24, 030901 (2019).

153. Zhanghao, K. et al. Super-resolution dipole orientation mapping via polarization demodulation. Light.: Sci. Appl. 5, e16166 (2016).

154. Zhanghao, K. et al. Super-resolution fluorescence polarization microscopy. J. Innovative Opt. Health Sci. 11, 1730002 (2018)

155. Chen, L. et al. Advances of super-resolution fluorescence polarization microscopy and its applications in life sciences. Comput. Struct. Biotechnol. J. 18, 2209-2216 (2020)

156. Wu, P. J. \& Walsh, J. T. Jr. Stokes polarimetry imaging of rat-tail tissue in a turbid medium using incident circularly polarized light. Lasers Surg. Med. 37, 396-406 (2005).

157. Macdonald, C. \& Meglinski, I. Backscattering of circular polarized light from a disperse random medium influenced by optical clearing. Laser Phys. Lett. 8, 324-328 (2011).

158. Qi, J. et al. Assessment of tissue polarimetric properties using Stokes polarimetric imaging with circularly polarized illumination. J. Biophotonics 11 e201700139 (2018)

159. Kunnen, B. et al. Application of circularly polarized light for non-invasive diagnosis of cancerous tissues and turbid tissue-like scattering media. J. Biophotonics 8, 317-323 (2015).

160. Xu, M. \& Alfano, R. R. Circular polarization memory of light. Phys. Rev. E 72, 065601 (2005)

161. Macdonald, C. M., Jacques, S. L. \& Meglinski, I. V. Circular polarization memory in polydisperse scattering media. Phys. Rev. E 91, 033204 (2015).

162. Wood, M. F. et al. Proof-of-principle demonstration of a Mueller matrix decomposition method for polarized light tissue characterization in vivo. J. Biomed. Opt. 14, 014029 (2009).

163. Wang, Y. et al. Mueller matrix microscope: a quantitative tool to facilitate detections and fibrosis scorings of liver cirrhosis and cancer tissues. J. Biomed. Opt. 21, 071112 (2016)

164. Liu, T. et al. Distinguishing structural features between Crohn's disease and gastrointestinal luminal tuberculosis using Mueller matrix derived parameters. J. Biophotonics 12, e201900151 (2019).

165. Shen, Y. X. et al. Comparative study of the influence of imaging resolution on linear retardance parameters derived from the Mueller matrix. Biomed. Opt. Express 12, 211-225 (2021).

166. Pierangelo, A. et al. Multispectral Mueller polarimetric imaging detecting residual cancer and cancer regression after neoadjuvant treatment for colorectal carcinomas. J. Biomed. Opt. 18, 046014 (2013).

167. Sun, M. H. et al. Characterizing the microstructures of biological tissues using Mueller matrix and transformed polarization parameters. Biomed. Opt. Express 5, 4223-4234 (2014).

168. Li, P. C. et al. Separating azimuthal orientation dependence in polarization measurements of anisotropic media. Opt. Express 26, 3791-3800 (2018).

169. Gil, J. J. Invariant quantities of a Mueller matrix under rotation and retarder transformations. J. Optical Soc. Am. A 33, 52-58 (2016).

170. Iqbal, M. et al. Comparative study of Mueller matrix transformation and polar decomposition for optical characterization of turbid media. Optik 224, 165508 (2020)

171. Sun, T. et al. Distinguishing anisotropy orientations originated from scattering and birefringence of turbid media using Mueller matrix derived parameters. Opt. Lett. 43, 4092-4095 (2018).

172. Khaliq, A. et al. Comparative study of $3 \times 3$ Mueller matrix transformation and polar decomposition. Opt. Commun. 485, 126756 (2021).

173. Tariq, A. et al. Physically realizable space for the purity-depolarization plane for polarized light scattering media. Phys. Rev. Lett. 119, 033202 (2017).

174. Swami, M. K. et al. Polar decomposition of $3 \times 3$ Mueller matrix: a tool for quantitative tissue polarimetry. Opt. Express 14, 9324-9337 (2006).

175. Wang, Y. F. et al. Study on the validity of $3 \times 3$ Mueller matrix decomposition. J. Biomed. Opt. 20, 065003 (2015).
176. Morio, J. \& Goudail, F. Influence of the order of diattenuator, retarder, and polarizer in polar decomposition of Mueller matrices. Opt. Lett. 29, 2234-2236 (2004).

177. Ghosh, N., Wood, M. F. G. \& Vitkin, I. A. Influence of the order of the constituent basis matrices on the Mueller matrix decomposition-derived polarization parameters in complex turbid media such as biological tissues. Opt. Commun. 283, 1200-1208 (2010).

178. Li, P. C. et al. Analysis of tissue microstructure with Mueller microscopy: logarithmic decomposition and Monte Carlo modeling. J. Biomed. Opt. 25 015002 (2020).

179. Li, P. C. et al. Characteristic Mueller matrices for direct assessment of the breaking of symmetries. Opt. Lett. 45, 706-709 (2020).

180. Vizet, J. et al. In vivo imaging of uterine cervix with a Mueller polarimetric colposcope. Sci. Rep. 7, 2471 (2017).

181. Zhanghao, K. et al. High-dimensional super-resolution imaging reveals heterogeneity and dynamics of subcellular lipid membranes. Nat. Commun. 11, 5890 (2020).

182. Zhanghao, K. et al. Super-resolution imaging of fluorescent dipoles via polarized structured illumination microscopy. Nat. Commun. 10, 4694 (2019).

183. Spandana, K. U., Mahato, K. K. \& Mazumder, N. Polarization-resolved Stokes-Mueller imaging: a review of technology and applications. Lasers Med. Sci. 34, 1283-1293 (2019).

184. Yang, B. et al. Polarized light spatial frequency domain imaging for nondestructive quantification of soft tissue fibrous structures. Biomed. Opt. Express 6, 1520-1533 (2015).

185. Clancy, N. T. et al. Polarised stereo endoscope and narrowband detection for minimal access surgery. Biomed. Opt. Express 5, 4108-4117 (2014).

186. Manhas, S. et al. Demonstration of full $4 \times 4$ Mueller polarimetry through an optical fiber for endoscopic applications. Opt. Express 23, 3047-3054 (2015).

187. Wood, T. C. \& Elson, D. S. Polarization response measurement and simulation of rigid endoscopes. Biomed. Opt. Express 1, 463-470 (2010).

188. Vizet, J. et al. Optical fiber-based full Mueller polarimeter for endoscopic imaging using a two-wavelength simultaneous measurement method. J. Biomed. Opt. 21, 071106 (2016).

189. Rivet, S., Bradu, A. \& Podoleanu, A. $70 \mathrm{kHz}$ full $4 \times 4$ Mueller polarimeter and simultaneous fiber calibration for endoscopic applications. Opt. Express 23, 23768-23786 (2015)

190. Forward, S. et al. Flexible polarimetric probe for $3 \times 3$ Mueller matrix measurements of biological tissue. Sci. Rep. 7, 11958 (2017).

191. Backman, V. et al. Polarized light scattering spectroscopy for quantitative measurement of epithelial cellular structures in situ. IEEE J. Sel. Top. Quantum Electron. 5, 1019-1026 (1999).

192. Chan, D. et al. In vivo spectroscopic ellipsometry measurements on human skin. J. Biomed. Opt. 12, 014023 (2007)

193. Banerjee, P. et al. Probing the fractal pattern and organization of Bacillus thuringiensis bacteria colonies growing under different conditions using quantitative spectral light scattering polarimetry. J. Biomed. Opt. 18, 035003 (2013).

194. Soni, J. et al. Quantitative fluorescence and elastic scattering tissue polarimetry using an Eigenvalue calibrated spectroscopic Mueller matrix system. Opt. Express 21, 15475-15489 (2013).

195. Jagtap, J. et al. Quantitative Mueller matrix fluorescence spectroscopy for precancer detection. Opt. Lett. 39, 243-246 (2014).

196. Satapathi, S., Soni, J. \& Ghosh, N. Fluorescent Mueller matrix analysis of a highly scattering turbid media. Appl. Phys. Lett. 104, 131902 (2014).

197. Huse, N., Schöenle, A. \& Hell, S. W. Z-polarized confocal microscopy. J. Biomed. Opt. 6, 273-276 (2001)

198. Lim, N. S. J. et al. Early detection of biomolecular changes in disrupted porcine cartilage using polarized Raman spectroscopy. J. Biomed. Opt. 16, 017003 (2011).

199. Ahlawat, S. et al. Polarized Raman spectroscopic investigations on hemoglobin ordering in red blood cells. J. Biomed. Opt. 19, 087002 (2014).

200. Chan, K. H. et al. Use of $2 \mathrm{D}$ images of depth and integrated reflectivity to represent the severity of demineralization in cross-polarization optical coherence tomography. J. Biophotonics 8, 36-45 (2015).

201. De Boer, J. F. \& Milner, T. E. Review of polarization sensitive optical coherence tomography and Stokes vector determination. J. Biomed. Opt. 7, 359-371 (2002).

202. Fan, C. M. \& Yao, G. Imaging myocardial fiber orientation using polarization sensitive optical coherence tomography. Biomed. Opt. Express 4, 460-465 (2013). 
203. Gladkova, N. et al. Evaluation of oral mucosa collagen condition with crosspolarization optical coherence tomography. J. Biophotonics 6, 321-329 (2013).

204. Hitzenberger, C. K. et al. Measurement and imaging of birefringence and optic axis orientation by phase resolved polarization sensitive optical coherence tomography. Opt. Express 9, 780-790 (2001).

205. Hong, Y. J. et al. Optically buffered Jones-matrix-based multifunctional optical coherence tomography with polarization mode dispersion correction. Biomed. Opt. Express 6, 225-243 (2015).

206. Jiao, S. L., Yao, G. \& Wang, L. V. Depth-resolved two-dimensional Stokes vectors of backscattered light and Mueller matrices of biological tissue measured with optical coherence tomography. Appl. Opt. 39, 6318-6324 (2000).

207. Kuranov, R. V. et al. Complementary use of cross-polarization and standard OCT for differential diagnosis of pathological tissues. Opt. Express 10, 707-713 (2002).

208. Lee, R. C. et al. Automated assessment of the remineralization of artificial enamel lesions with polarization-sensitive optical coherence tomography. Biomed. Opt. Express 5, 2950-2962 (2014).

209. Popescu, D. P. et al. Assessment of early demineralization in teeth using the signal attenuation in optical coherence tomography images. J. Biomed. Opt. 13, 054053 (2008).

210. Sugita, M. et al. Retinal nerve fiber bundle tracing and analysis in human eye by polarization sensitive OCT. Biomed. Opt. Express 6, 1030-1054 (2015).

211. Ugryumova, N. et al. The collagen structure of equine articular cartilage, characterized using polarization-sensitive optical coherence tomography. J. Phys. D: Appl. Phys. 38, 2612-2619 (2005).

212. Vitkin, A., Ghosh, N. \& De Martino, A. Tissue polarimetry. Photonics.: Sci. Found. Technol. Appl. 4, 239-321 (2015).

213. Tuchin, V. V., Wang, L. V. \& Zimnyakov, D. A. Optical Polarization in Biomedical Applications (Springer, 2006)

214. Wang, L. V., Coté, G. L. \& Jacques, S. L. Special section guest editorial: tissue polarimetry. J. Biomed. Opt. 7, 278 (2002)

215. Wang, L. V. \& Wu, H. I. Biomedical Optics: Principles and Imaging (John Wiley \& Sons, 2009).

216. Wang, S. \& Larin, K. V. Optical coherence elastography for tissue characterization: a review. J. Biophotonics 8, 279-302 (2015).

217. Yamanari, M. et al. Scleral birefringence as measured by polarization-sensitive optical coherence tomography and ocular biometric parameters of human eyes in vivo. Biomed. Opt. Express 5, 1391-1402 (2014).

218. Yao, G. \& Wang, L. V. Two-dimensional depth-resolved Mueller matrix characterization of biological tissue by optical coherence tomography. Opt. Lett. 24, 537-539 (1999)

219. Milione, G. et al. Measuring the self-healing of the spatially inhomogeneous states of polarization of vector Bessel beams. J. Opt. 17, 035617 (2015)

220. Mansfield, J. C. et al. Collagen fiber arrangement in normal and diseased cartilage studied by polarization sensitive nonlinear microscopy. J. Biomed. Opt. 13, 044020 (2008).

221. Tanaka, Y. et al. Motion-artifact-robust, polarization-resolved secondharmonic-generation microscopy based on rapid polarization switching with electro-optic Pockells cell and its application to in vivo visualization of collagen fiber orientation in human facial skin. Biomed. Opt. Express $\mathbf{5}$, 1099-1113 (2014).

222. DeWalt, E. L. et al. Polarization-modulated second harmonic generation ellipsometric microscopy at video rate. Anal. Chem. 86, 8448-8456 (2014).

223. Pavone, F. S. \& Campagnola, P. J. Second Harmonic Generation Imaging (CRC Press, 2013).

224. Brasselet, S. Polarization-resolved nonlinear microscopy: application to structural molecular and biological imaging. Adv. Opt. Photonics 3, 205-271 (2011).

225. Kapsokalyvas, D. et al. In-vivo imaging of psoriatic lesions with polarization multispectral dermoscopy and multiphoton microscopy. Biomed. Opt. Express 5, 2405-2419 (2014).

226. Golaraei, A. et al. Characterization of collagen in non-small cell lung carcinoma with second harmonic polarization microscopy. Biomed. Opt. Express $\mathbf{5}$, 3562-3567 (2014).

227. Daly, S. M. \& Leahy, M. J. 'Go with the flow: a review of methods and advancements in blood flow imaging. J. Biophotonics 6, 217-255 (2013).

228. Wolf, E. Introduction to the Theory of Coherence and Polarization of Light (Cambridge University Press, 2007).
229. Abrahamsson, S. et al. MultiFocus polarization microscope (MF-PolScope) for 3D polarization imaging of up to 25 focal planes simultaneously. Opt. Express 23, 7734-7754 (2015).

230. Chen, D. S. et al. Study of optical clearing in polarization measurements by Monte Carlo simulations with anisotropic tissue-mimicking models. J. Biomed. Opt. 21, 081209 (2016).

231. Wang, Y. et al. Differentiating characteristic microstructural features of cancerous tissues using Mueller matrix microscope. Micron 79, 8-15 (2015).

232. Hafi, N. et al. Fluorescence nanoscopy by polarization modulation and polarization angle narrowing. Nat. Methods 11, 579-584 (2014).

233. Cruz, C. A. V. et al. Quantitative nanoscale imaging of orientational order in biological filaments by polarized superresolution microscopy. Proc. Natl Acad. Sci. USA 113, E820-E828 (2016).

234. Kampmann, M. et al. Mapping the orientation of nuclear pore proteins in living cells with polarized fluorescence microscopy. Nat. Struct. Mol. Biol. 18, 643-649 (2011).

235. Sase, I. et al. Axial rotation of sliding actin filaments revealed by singlefluorophore imaging. Proc. Natl Acad. Sci. USA 94, 5646-5650 (1997).

236. Forkey, J. N. et al. Three-dimensional structural dynamics of myosin $\mathrm{V}$ by single-molecule fluorescence polarization. Nature 422, 399-404 (2003).

237. Sosa, $H$. et al. ADP-induced rocking of the kinesin motor domain revealed by single-molecule fluorescence polarization microscopy. Nat. Struct. Biol. 8, 540-544 (2001).

238. DeMay, B. S. et al. Septin filaments exhibit a dynamic, paired organization that is conserved from yeast to mammals. J. Cell Biol. 193, 1065-1081 (2011).

239. DeMay, B. S. et al. Rapid and quantitative imaging of excitation polarized fluorescence reveals ordered septin dynamics in live yeast. Biophys. J. 101, 985-994 (2011).

240. Axelrod, D. Carbocyanine dye orientation in red cell membrane studied by microscopic fluorescence polarization. Biophys. J. 26, 557-573 (1979).

241. Schütz, G. J., Schindler, H. \& Schmidt, T. Imaging single-molecule dichroism. Opt. Lett. 22, 651-653 (1997).

242. Du, E. et al. Mueller matrix polarimetry for differentiating characteristic features of cancerous tissues. J. Biomed. Opt. 19, 076013 (2014).

243. Pierangelo, A. et al. Polarimetric imaging of uterine cervix: a case study. Opt Express 21, 14120-14130 (2013).

244. Rehbinder, J. et al. Ex vivo Mueller polarimetric imaging of the uterine cervix: a first statistical evaluation. J. Biomed. Opt. 21, 071113 (2016).

245. Shukla, P. \& Pradhan, A. Mueller decomposition images for cervical tissue: potential for discriminating normal and dysplastic states. Opt. Express 17, 1600-1609 (2009).

246. Chue-Sang, J. et al. Use of combined polarization-sensitive optical coherence tomography and Mueller matrix imaging for the polarimetric characterization of excised biological tissue. J. Biomed. Opt. 21, 071109 (2016).

247. Novikova, T. et al. The origins of polarimetric image contrast between healthy and cancerous human colon tissue. Appl. Phys. Lett. 102, 241103 (2013).

248. Ahmad, I. et al. Ex vivo characterization of normal and adenocarcinoma colon samples by Mueller matrix polarimetry. J. Biomed. Opt. 20, 056012 (2015).

249. Pierangelo, A. et al. Ex-vivo characterization of human colon cancer by Mueller polarimetric imaging. Opt. Express 19, 1582-1593 (2011).

250. Pierangelo, A. et al. Ex vivo photometric and polarimetric multilayer characterization of human healthy colon by multispectral Mueller imaging. J. Biomed. Opt. 17, 066009 (2012).

251. Dubreuil, M. et al. Mueller matrix polarimetry for improved liver fibrosis diagnosis. Opt. Lett. 37, 1061-1063 (2012).

252. Wang, W. F. et al. Roles of linear and circular polarization properties and effect of wavelength choice on differentiation between ex vivo normal and cancerous gastric samples. J. Biomed. Opt. 19, 046020 (2014).

253. Borovkova, M. et al. Evaluating $\beta$-amyloidosis progression in Alzheimer's disease with Mueller polarimetry. Biomed. Opt. Express 11, 4509-4519 (2020).

254. Alali, S. et al. Assessment of local structural disorders of the bladder wall in partial bladder outlet obstruction using polarized light imaging. Biomed. Opt Express 5, 621-629 (2014).

255. Backman, V. et al. Detection of preinvasive cancer cells. Nature 406, 35-36 (2000).

256. Qi, J. \& Elson, D. S. A high definition Mueller polarimetric endoscope for tissue characterisation. Sci. Rep. 6, 25953 (2016).

257. Gan, Y. \& Fleming, C. P. Extracting three-dimensional orientation and tractography of myofibers using optical coherence tomography. Biomed. Opt. Express 4, 2150-2165 (2013). 
258. Pham, H. T. T. et al. Optical parameters of human blood plasma, collagen, and calfskin based on the Stokes-Mueller technique. Appl. Opt. 57, 4353-4359 (2018).

259. Lu, R. W. et al. A polarization-sensitive light field imager for multi-channel angular spectroscopy of light scattering in biological tissues. Quant. Imaging Med. Surg. 5, 1-8 (2015).

260. Ghosh, N. et al. Mueller matrix decomposition for polarized light assessment of biological tissues. J. Biophotonics 2, 145-156 (2009).

261. Wood, M. F. G. et al. Polarization birefringence measurements for characterizing the myocardium, including healthy, infarcted, and stem-cellregenerated tissues. J. Biomed. Opt. 15, 047009 (2010).

262. Ahmad, I. et al. Polarimetric assessment of healthy and radiofrequency ablated porcine myocardial tissue. J. Biophotonics 9, 750-759 (2016).

263. He, H. H. et al. Monitoring microstructural variations of fresh skeletal muscle tissues by Mueller matrix imaging. J. Biophotonics 10,664-673 (2017).

264. Sugita, S. \& Matsumoto, T. Quantitative measurement of the distribution and alignment of collagen fibers in unfixed aortic tissues. J. Biomech. 46, 1403-1407 (2013).

265. Saytashev, I. et al. Self validating Mueller matrix Micro-Mesoscope (SAMMM) for the characterization of biological media. Opt. Lett. 45, 2168-2171 (2020).

266. Chen, Z. H. et al. A collinear reflection Mueller matrix microscope for backscattering Mueller matrix imaging. Opt. Lasers Eng. 129, 106055 (2020).

267. Gonzalez, M. et al. Design and implementation of a portable colposcope Mueller matrix polarimeter. J. Biomed. Opt. 25, 116006 (2020).

268. Zotter, S. et al. Measuring retinal nerve fiber layer birefringence, retardation, and thickness using wide-field, high-speed polarization sensitive spectral domain OCT. Investigative Ophthalmol. Vis. Sci. 54, 72-84 (2013).

269. Dong, Y. et al. A quantitative and non-contact technique to characterise microstructural variations of skin tissues during photo-damaging process based on Mueller matrix polarimetry. Sci. Rep. 7, 14702 (2017).

270. Liu, X. Y. et al. Tissue-like phantoms for quantitative birefringence imaging. Biomed. Opt. Express 8, 4454-4465 (2017).

271. Swami, M. K. et al. Effect of gold nanoparticles on depolarization characteristics of Intralipid tissue phantom. Opt. Lett. 38, 2855-2857 (2013).

272. Guo, Y. H. et al. Study on retardance due to well-ordered birefringent cylinders in anisotropic scattering media. J. Biomed. Opt. 19, 065001 (2014).

273. Li, X. P. et al. Polarimetric learning: a Siamese approach to learning distance metrics of algal Mueller matrix images. Appl. Opt. 57, 3829-3837 (2018).
274. Heinrich, $C$. et al. Mueller polarimetric imaging of biological tissues: classification in a decision-theoretic framework. J. Opt. Soc. Am. A 35, 2046-2057 (2018).

275. Wetzstein, G. et al. Inference in artificial intelligence with deep optics and photonics. Nature 588, 39-47 (2020).

276. Rivenson, Y. et al. Deep learning microscopy. Optica 4, 1437-1443 (2017).

277. He, C. et al. Vectorial adaptive optics: correction of polarization and phase. in Adaptive Optics and Wavefront Control for Biological Systems VI. Vol. 11248 (eds Bifano, T. G., Gigan, S., \& Ji, N.) (International Society for Optics and Photonics, 2020). https://www.spiedigitallibrary.org/conference-proceedingsof-spie/11248/1124808/Nectorial-adaptive-opticscorrection-of-polarizationand-phase/10.1117/12.2547715.full?SSO=1.

278. $\mathrm{Hu}, \mathrm{Q}$. et al. Arbitrary vectorial state conversion using liquid crystal spatial light modulators. Opt. Commun. 459, 125028 (2020).

279. $\mathrm{Hu}, \mathrm{Q} ., \mathrm{He}, \mathrm{C} . \&$ Booth, M. J. Arbitrary complex retarders using a sequence of spatial light modulators as the basis for adaptive polarisation compensation. J. Opt. 23, 065602 (2021).

280. Dai, Y. Y. et al. Active compensation of extrinsic polarization errors using adaptive optics. Opt. Express 27, 35797-35810 (2019).

281. Rubin, N. A. et al. Matrix Fourier optics enables a compact full-Stokes polarization camera. Science 365, eaax1839 (2019).

282. Dorrah, A. H. et al. Metasurface optics for on-demand polarization transformations along the optical path. Nat. Photonics 15, 287-296 (2021).

283. Pan, T. et al. Biophotonic probes for bio-detection and imaging. Light.: Sci. Appl. 10, 124 (2021).

284. Samim, M., Krouglov, S. \& Barzda, V. Nonlinear Stokes-Mueller polarimetry. Phys. Rev. A 93, 013847 (2016).

285. Kontenis, L. et al. in CLEO: Science and Innovations (Optical Society of America, 2016).

286. Okoro, C. Second-harmonic Generation-based Mueller Matrix Polarization Analysis Of Collagen-rich Tissues. PhD thesis (University of Illinois, UrbanaChampaign, Urbana, 2018).

287. Krouglov, S. \& Barzda, V. Three-dimensional nonlinear Stokes-Mueller polarimetry. J. Opt. Soc. Am. B 36, 541-550 (2019).

288. Azzam, R. M. A. Arrangement of four photodetectors for measuring the state of polarization of light. Opt. Lett. 10, 309-311 (1985).

289. Mehta, S. B. et al. Dissection of molecular assembly dynamics by tracking orientation and position of single molecules in live cells. Proc. Natl Acad. Sci. USA 113, E6352-E6361 (2016). 